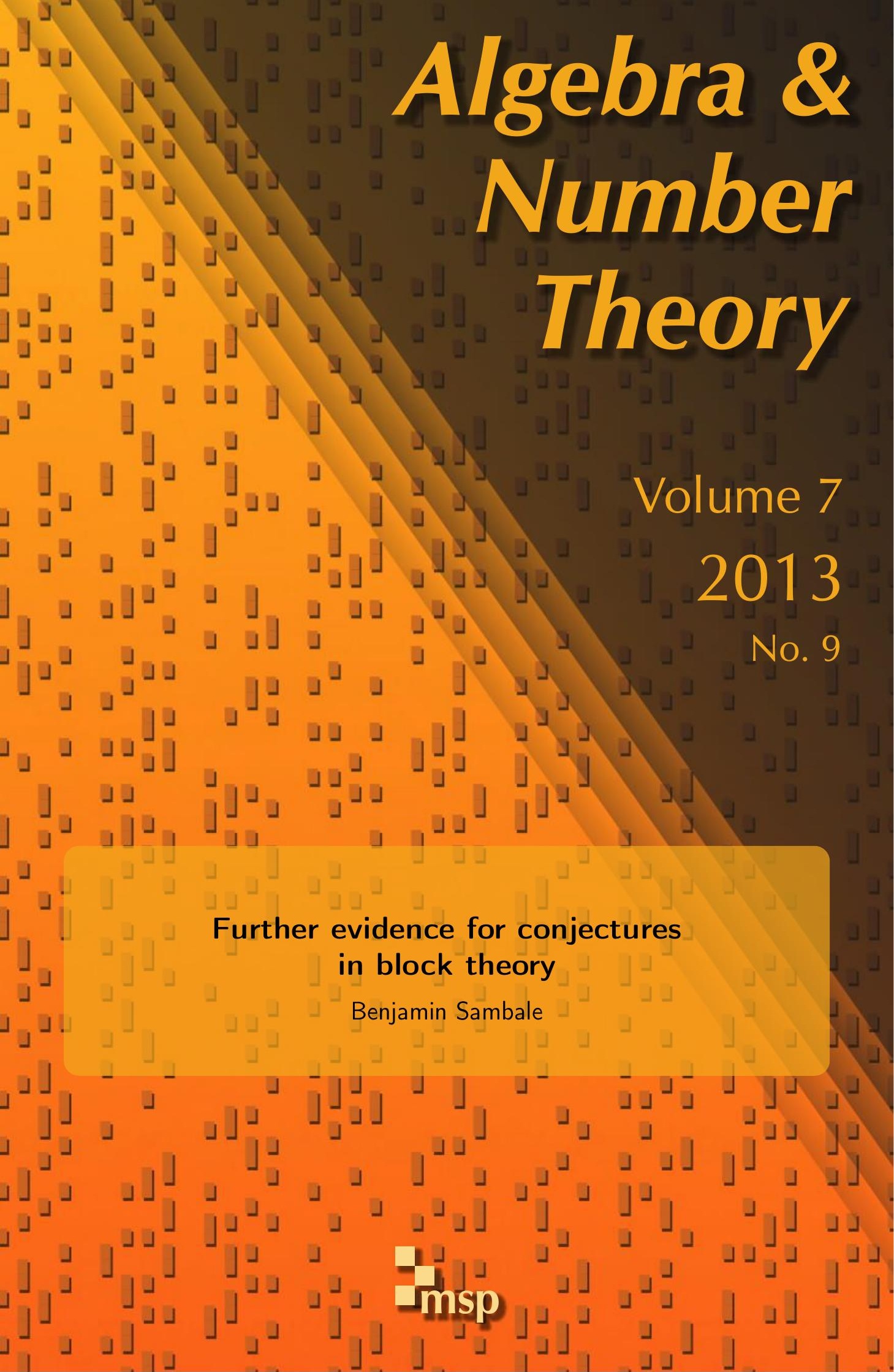




\title{
Further evidence for conjectures in block theory
}

\author{
Benjamin Sambale
}

\begin{abstract}
We prove new inequalities concerning Brauer's $k(B)$-conjecture and Olsson's conjecture by generalizing old results. After that, we obtain the invariants for 2-blocks of finite groups with certain bicyclic defect groups. Here, a bicyclic group is a product of two cyclic subgroups. This provides an application for the classification of the corresponding fusion systems in a previous paper. To some extent, this generalizes previously known cases with defect groups of types $D_{2^{n}} \times C_{2^{m}}, Q_{2^{n}} \times C_{2^{m}}$ and $D_{2^{n}} * C_{2^{m}}$. As a consequence, we prove Alperin's weight conjecture and other conjectures for several new infinite families of nonnilpotent blocks. We also prove Brauer's $k(B)$-conjecture and Olsson's conjecture for the 2-blocks of defect at most 5. This completes results from a previous paper. The $k(B)$-conjecture is also verified for defect groups with a cyclic subgroup of index at most 4 . Finally, we consider Olsson's conjecture for certain 3-blocks.
\end{abstract}

\section{Introduction}

Let $B$ be a $p$-block of a finite group $G$. One aim of this paper is to establish new inequalities on the number of irreducible characters of $B$ in terms of subsections. We outline the idea behind these things.

Olsson [1981] proved the following:

$$
l(B) \leq 2 \Longrightarrow k(B) \leq p^{d},
$$

where $d$ is the defect of $B$. In particular, this gives an example for Brauer's $k(B)$ conjecture. However, in practice this implication is not so useful because usually the knowledge of $l(B)$ already implies the exact value of $k(B)$. Since the proofs in [Olsson 1981] only rely on computations with the contributions of the trivial subsection $(1, B)$, it seems likely that one can extend this result to major subsections. Then we would be able to apply induction on $d$ (see Theorem 4.9). Hence, let $\left(z, b_{z}\right)$ be a major subsection such that $l\left(b_{z}\right) \leq 2$. In case $l\left(b_{z}\right)=1$, we have

Keywords: 2-blocks, bicyclic defect groups, Brauer's $k(B)$-conjecture, Alperin's weight conjecture. 
$\sum k_{i}(B) p^{2 i} \leq p^{d}$ by [Robinson 1991, Theorem 3.4] (a stronger bound can be found in [Héthelyi et al. 2013]).

In Section 2, we show

$$
l\left(b_{z}\right) \leq 2 \Rightarrow k(B) \leq p^{d} .
$$

In contrast to Olsson's paper, we use methods from [Robinson 1991] and [Sambale 2011b]. For $p=2$, Olsson proved the stronger statement $l(B) \leq 3 \Rightarrow k(B) \leq p^{d}$. Using his ideas, we generalize this to major subsections as well. The underlying properties of the contribution matrices were first discovered by Brauer [1968], but we will refer to [Feit 1982] for a more accessible account. Using Galois theory, we overcome the difficulty that the contributions are not necessarily integers in this general setting.

More generally, we consider arbitrary subsections for the prime 2 in order to give bounds on the number of characters of height 0 . Here it is known by [Broué 1980] (more recent accounts can be found in [Robinson 1992; Murai 2000]) that the corresponding contributions for characters of height 0 do not vanish. Using exactly the same method, we show that $k_{0}(B) \leq 2^{q}$ if there is a subsection $\left(u, b_{u}\right)$ such that $b_{u}$ has defect $q$ and $l\left(b_{u}\right) \leq 3$.

In Section 3, we present new infinite families of defect groups for which the block invariants can be calculated. These defect groups are examples of bicyclic 2-groups (i.e., $D=\langle x\rangle\langle y\rangle$ for some $x, y \in D$ ). The proofs make use of the classification of the corresponding fusion systems in [Sambale 2012b]. However, we cannot handle all bicyclic 2-groups. We also remark that these defect groups are in a sense noncommutative versions of the groups $D_{2^{n}} \times C_{2^{m}}, Q_{2^{n}} \times C_{2^{m}}$ and $D_{2^{n}} * C_{2^{m}}$ covered in [Sambale 2012a; 2013b; 2013a]. As a consequence, we verify numerous conjectures including Alperin's weight conjecture for these blocks.

In Section 4, we collect some more or less related examples for block invariants. In particular, we discuss some defect groups of order 32. One of the main results here is the verification of Brauer's $k(B)$-conjecture for the 2-blocks of defect at most 5. This completes [Sambale 2011c, Theorem 3]. The new ingredient here is in fact an old result of Brauer that uses the inverse of the Cartan matrix of a major subsection.

In Section 5, we obtain new cases for Olsson's conjecture. In particular, we handle the 2-blocks of defect at most 5 and some 3-blocks with defect group of 3-rank 2 that were left over in [Héthelyi et al. 2013].

\section{New inequalities}

Let $B$ be a $p$-block of a finite group $G$ with defect group $D$. We define the height $h(\chi)$ of a character $\chi \in \operatorname{Irr}(B)$ by $\chi(1)_{p}=p^{h(\chi)}|G: D|_{p}$. Moreover, 
$\operatorname{Irr}_{i}(B):=\{\chi \in \operatorname{Irr}(B): h(\chi)=i\}, k(B):=|\operatorname{Irr}(B)|$ and $k_{i}(B):=\left|\operatorname{Irr}_{i}(B)\right|$ for $i \geq 0$. As usual, we denote the set of irreducible Brauer characters of $B$ by $\operatorname{IBr}(B)$ and its cardinality by $l(B):=|\operatorname{IBr}(B)|$.

In the following, we choose an element $z \in \mathrm{Z}(D)$. Then there exists a Brauer correspondent $b_{z}$ of $B$ in $\mathrm{C}_{G}(z)$. The pair $\left(z, b_{z}\right)$ is called a major subsection.

Theorem 2.1. Let $B$ be a p-block of a finite group with defect $d$, and let $\left(z, b_{z}\right)$ be a major subsection such that $l\left(b_{z}\right) \leq 2$. Then one of the following holds:

$$
\begin{gathered}
\sum_{i=0}^{\infty} k_{i}(B) p^{2 i} \leq p^{d} . \\
k(B) \leq \begin{cases}((p+3) / 2) p^{d-1} & \text { if } p>2, \\
\frac{2}{3} 2^{d} & \text { if } p=2 .\end{cases}
\end{gathered}
$$

In particular, Brauer's $k(B)$-conjecture holds for $B$.

Proof. In case $l\left(b_{z}\right)=1$, equation (1) holds. Hence, let $l\left(b_{z}\right)=2$, and let $C_{z}=\left(c_{i j}\right)$ be the Cartan matrix of $b_{z}$ up to basic sets. We consider the number

$$
q\left(b_{z}\right):=\min \left\{x p^{d} C_{z}^{-1} x^{\mathrm{T}}: 0 \neq x \in \mathbb{Z}^{l\left(b_{z}\right)}\right\} \in \mathbb{N} .
$$

If $q\left(b_{z}\right)=1$, equation (1) follows from [Robinson 1991, Theorem 3.4.1]. Therefore, we may assume $q\left(b_{z}\right) \geq 2$. Then Brauer's $k(B)$-conjecture already holds by [Feit 1982, Theorem V.9.17], but we want to obtain the stronger bound (2). Since $p^{d}$ is always an elementary divisor of $C_{z}$, we see that $C_{z}$ is not a diagonal matrix. This allows us to apply [Héthelyi et al. 2013, Theorem 2.4]. All entries of $C_{z}$ are divisible by the smallest elementary divisor $\gamma:=p^{-d} \operatorname{det} C_{z}$. Hence, we may consider the integral matrix $\widetilde{C}_{z}=\left(\tilde{c}_{i j}\right):=\gamma^{-1} C_{z}$. After changing the basic set, we may assume that $0<2 \tilde{c}_{12} \leq \tilde{c}_{11} \leq \tilde{c}_{22}$. Then

$$
\tilde{c}_{11} \tilde{c}_{22}-\frac{\tilde{c}_{11}^{2}}{4} \leq \tilde{c}_{11} \tilde{c}_{22}-\tilde{c}_{12}^{2}=\operatorname{det} \widetilde{C}_{z}=\frac{p^{d}}{\gamma}
$$

and

$$
\tilde{c}_{11}+\tilde{c}_{22} \leq \frac{5}{4} \tilde{c}_{11}+\frac{\operatorname{det} \widetilde{C}_{z}}{\tilde{c}_{11}}=: f\left(\tilde{c}_{11}\right) .
$$

A discussion of the convex function $f\left(\tilde{c}_{11}\right)$ as in [Sambale 2011b, Theorem 1] shows that $\tilde{c}_{11}+\tilde{c}_{22} \leq f(2)$. Now [Héthelyi et al. 2013, Theorem 2.4] leads to

$$
k(B) \leq \gamma\left(\tilde{c}_{11}+\tilde{c}_{22}-\tilde{c}_{12}\right) \leq \gamma(f(2)-1) \leq \frac{p^{d}+3 \gamma}{2} .
$$

Since $\gamma \leq p^{d-1}$, we get (2) for $p$ odd. In order to deduce the $k(B)$-conjecture, we need to consider the case $p=2$. If $\tilde{c}_{11}=2$, we must have $\tilde{c}_{12}=1$. Hence, under 
these circumstances $p>2$ since otherwise $\operatorname{det} \widetilde{C}_{z}$ is not a $p$-power. Now assume $\tilde{c}_{11} \geq 3$ and $p=2$. Since

$$
p^{d} C_{z}^{-1}=\frac{p^{d}}{\gamma} \widetilde{C}_{z}^{-1}=\left(\begin{array}{cc}
\tilde{c}_{22} & -\tilde{c}_{12} \\
-\tilde{c}_{12} & \tilde{c}_{11}
\end{array}\right)
$$

we have $q\left(b_{z}\right) \geq 3$. Now [Feit 1982, Theorem V.9.17] implies (2). We will derive another estimation for $p=2$ in Theorem 2.2 below.

It is conjectured that the matrix $C_{z}$ for $l\left(b_{z}\right) \geq 2$ in the proof of Theorem 2.1 cannot have diagonal shape (this holds for $p$-solvable groups by [Sambale 2011b, Lemma 1]). Hence, for $l\left(b_{z}\right)=2$ Theorem 2.1(2) might always apply. Then $k(B)<p^{d}$ unless $p=3$.

In order to improve Theorem 2.1 for $p=2$, we need more notation. Suppose as before that $\left(z, b_{z}\right)$ is a major subsection. We denote the corresponding part of the generalized decomposition matrix by $D_{z}:=\left(d_{\chi \chi}^{z}: \chi \in \operatorname{Irr}(B), \varphi \in \operatorname{IBr}\left(b_{z}\right)\right)$. Then the Cartan matrix of $b_{z}$ is given by $C_{z}:=D_{z}^{\mathrm{T}} \frac{\chi}{D_{z}}$. Moreover, the contribution matrix of $b_{z}$ is defined as

$$
M_{z}:=\left(m_{\chi \psi}^{z}\right)_{\chi, \psi \in \operatorname{Irr}(B)}=|D| D_{z} C_{z}^{-1} \bar{D}_{z}^{\mathrm{T}} .
$$

In case $|\langle z\rangle| \leq 2$, it can be seen easily that $M_{z}$ is an integral matrix. Then most proofs of [Olsson 1981] remain true without any changes. This was more or less done in [Robinson 2008] (compare also Corollary 3.5 in [Robinson 1991]). In the general case, we have to put a bit more effort into the proof.

Theorem 2.2. Let $B$ be a 2-block of a finite group with defect $d$, and let $\left(z, b_{z}\right)$ be a major $B$-subsection such that $l\left(b_{z}\right) \leq 3$. Then

$$
k(B) \leq k_{0}(B)+\frac{2}{3} \sum_{i=1}^{\infty} 2^{i} k_{i}(B) \leq 2^{d} .
$$

In particular, Brauer's $k(B)$-conjecture is satisfied for $B$.

Proof. Observe that by construction $m_{\chi \chi}^{z}$ is a positive real number for every $\chi \in \operatorname{Irr}(B)$ since $C_{z}$ is positive definite. Since all elementary divisors of $C_{z}$ are divisors of $2^{d}$, the matrix $2^{d} C_{z}^{-1}$ is integral. In particular, the numbers $m_{\chi \psi}^{z}$ are also algebraic integers. Let $\chi \in \operatorname{Irr}(B)$ be a character of height 0 . Let $|\langle z\rangle|=2^{n}$. In case $n \leq 1$, the proof is much easier. For this reason, we assume $n \geq 2$. We write

$$
m_{\chi \chi}^{z}=\sum_{j=0}^{2^{n-1}-1} a_{j}(\chi) \zeta^{j}
$$

with $\zeta:=e^{2 \pi i / 2^{n}}$ and $a_{j}(\chi) \in \mathbb{Z}$ for $j=0, \ldots, 2^{n-1}-1$. As usual, the Galois group $\mathscr{G}$ of the $2^{n}$-th cyclotomic field acts on $\operatorname{Irr}(B)$, on the rows of $D_{z}$ and thus 
also on $M_{z}$ in an obvious manner. Let $\Gamma$ be the orbit of $\chi$ under $\mathscr{G}$. Set $m:=|\Gamma|$. Then we have

$$
m a_{0}(\chi)=\sum_{\psi \in \Gamma} m_{\psi \psi}^{z}>0
$$

Assume first that $a_{0}(\chi)=1$. Since $M_{z}^{2}=M_{z} \bar{M}_{z}^{\mathrm{T}}=2^{d} M_{z}$ (see [Feit 1982, Theorem V.9.4]), it follows that

$$
m 2^{d}=\sum_{\substack{\psi \in \Gamma \\ \tau \in \operatorname{Irr}(B)}}\left|m_{\psi \tau}^{z}\right|^{2} .
$$

Applying Galois theory gives

$$
\prod_{\substack{\psi \in \Gamma \\ \tau \in \operatorname{Irr}_{i}(B)}}\left|m_{\psi \tau}^{z}\right|^{2} \in \mathbb{Q}
$$

for all $i \geq 0$. By [Feit 1982, Theorem V.9.4], we also know $v\left(m_{\psi \tau}^{z}\right)=h(\tau)$, where $v$ is the 2 -adic valuation and $\psi \in \Gamma$. Hence, also the numbers $m_{\psi \tau}^{z} 2^{-h(\tau)}$ are algebraic integers. This implies

$$
\mathbb{Z} \ni \prod_{\substack{\psi \in \Gamma \\ \tau \in \operatorname{Irr}_{i}(B)}} p^{-2 i}\left|m_{\psi \tau}^{z}\right|^{2} \geq 1 .
$$

Now using the inequality of arithmetic and geometric means, we obtain

$$
\sum_{\substack{\psi \in \Gamma \\ \tau \in \operatorname{Irr}_{i}(B)}}\left|m_{\psi \tau}^{z}\right|^{2} \geq m 2^{2 i} k_{i}(B)
$$

for all $i \geq 0$. Summing over $i$ gives

$$
m 2^{d}=\sum_{\substack{\psi \in \Gamma \\ \tau \in \operatorname{Irr}(B)}}\left|m_{\psi \tau}^{z}\right|^{2} \geq m \sum_{i=0}^{\infty} 2^{2 i} k_{i}(B),
$$

which is even more than we wanted to prove.

Hence, we can assume that $a_{0}(\chi) \geq 2$ for all $\chi \in \operatorname{Irr}(B)$ such that $h(\chi)=0$. It is well-known that the ring of integers of $\mathbb{Q}(\zeta) \cap \mathbb{R}$ has basis $\left\{1, \zeta^{j}+\zeta^{-j}=\right.$ $\left.\zeta^{j}-\zeta^{2^{n-1}-j}: j=1, \ldots, 2^{n-2}-1\right\}$. In particular, the numbers $a_{j}(\chi)$ for $j \geq 1$ come in pairs modulo 2. Since $v\left(m_{\chi \chi}^{z}\right)=0$, we even have $a_{0}(\chi) \geq 3$. For an arbitrary character $\psi \in \operatorname{Irr}(B)$ of positive height, we already know that $m_{\psi \psi}^{z} 2^{-h(\psi)}$ is a positive algebraic integer. Hence, $2^{h(\psi)} \mid a_{j}(\psi)$ for all $j \geq 0$. By [Feit 1982, Theorem V.9.4], we have $v\left(m_{\psi \psi}^{z}\right)>h(\psi)$. Thus, we even have $2^{h(\psi)+1} \mid a_{0}(\psi)$. As above, we also have $a_{0}(\psi)>0$. This implies $\sum_{\psi \in \operatorname{Ir}_{i}(B)} m_{\psi \psi}^{z} \geq 2^{i+1} k_{i}(B)$ for $i \geq 1$ 
via Galois theory. Using $\operatorname{tr} M_{z}=2^{d} l\left(b_{z}\right)$, it follows that

$$
3 \cdot 2^{d} \geq \sum_{\psi \in \operatorname{Irr}(B)} m_{\psi \psi}^{z} \geq 3 k_{0}(B)+\sum_{i=1}^{\infty} 2^{i+1} k_{i}(B) .
$$

This proves the claim.

We remark that Theorem 6(ii) in [Olsson 1981] should read $l(B) \leq p^{2}-1$ (compare with Theorem 6*(ii)).

It is easy to see that the proof of Theorem 2.2 can be generalized to the following:

Proposition 2.3. Let $B$ be a 2-block of a finite group with defect $d$, and let $\left(z, b_{z}\right)$ be a major $B$-subsection. Then for every odd number $\alpha$ one of the following holds:

$$
\begin{gathered}
\sum_{i=0}^{\infty} 2^{2 i} k_{i}(B) \leq 2^{d} \alpha . \\
(\alpha+2) k_{0}(B)+\sum_{i=1}^{\infty} 2^{i+1} k_{i}(B) \leq 2^{d} l\left(b_{z}\right) .
\end{gathered}
$$

Proof. As in Theorem 2.2, let $\chi \in \operatorname{Irr}_{0}(B)$ and define $a_{0}(\chi)$ similarly. In case $a_{0}(\chi) \leq \alpha$, the first inequality applies. Otherwise, the second inequality applies.

Observe that Proposition 2.3 also covers (a generalization of) [Olsson 1981, Theorem 8] for $p=2$.

Going over to arbitrary subsections (i.e., the element does not necessarily belong to $\mathrm{Z}(D)$ ), we can prove the following result concerning Olsson's conjecture. This improves [Robinson 1992, Theorem 3.1] for $p=2$.

Theorem 2.4. Let $B$ be a 2-block of a finite group, and let $\left(u, b_{u}\right)$ be a $B$-subsection such that $b_{u}$ has defect $q$. Set $\alpha:=\left\lfloor\sqrt{l\left(b_{u}\right)}\right\rfloor$ if $\left\lfloor\sqrt{l\left(b_{u}\right)}\right\rfloor$ is odd and $\alpha:=$ $l\left(b_{u}\right) /\left(\left\lfloor\sqrt{l\left(b_{u}\right)}\right\rfloor+1\right)$ otherwise. Then $k_{0}(B) \leq \alpha 2^{q}$. In particular, $k_{0}(B) \leq 2^{q}$ if $l\left(b_{u}\right) \leq 3$.

Proof. The contributions for $\left(u, b_{u}\right)$ are defined by

$$
M_{u}:=\left(m_{\chi \psi}^{u}\right)_{\chi, \psi \in \operatorname{Irr}(B)}=p^{q} D_{u} C_{u}^{-1}{\overline{D_{u}}}^{\mathrm{T}} .
$$

By [Murai 2000, Corollary 1.15], we still have $m_{\chi \psi}^{u} \neq 0$ as long as $h(\chi)=h(\psi)=0$. However, in all other cases it is possible that $m_{\chi \psi}^{u}=0$. So we can copy the proof of Theorem 2.2 by leaving out the characters of positive height. This gives $k_{0}(B) \leq \alpha 2^{q}$ or $k_{0}(B) \leq 2^{q} l\left(b_{u}\right) /(\alpha+2)$ for every odd number $\alpha$. If $\left\lfloor\sqrt{l\left(b_{u}\right)}\right\rfloor$ is odd, we choose $\alpha:=\left\lfloor\sqrt{l\left(b_{u}\right)}\right\rfloor$. Otherwise, we take $\alpha:=\left\lfloor\sqrt{l\left(b_{u}\right)}\right\rfloor-1$. The result follows.

Finally, we generalize the "dual" inequalities in [Olsson 1981]. For this, let $M_{z}^{\prime}:=\left(m_{\chi \psi}^{\prime}\right)=2^{d} 1_{k(B)}-M_{z}$. 
Proposition 2.5. Let $B$ be a 2-block of a finite group with defect $d$, and let $\left(z, b_{z}\right)$ be a major B-subsection. Then for every odd number $\alpha$ one of the following holds:

$$
\begin{gathered}
\sum_{i=0}^{\infty} 2^{2 i} k_{i}(B) \leq 2^{d} \alpha . \\
(\alpha+2) k_{0}(B)+\sum_{i=1}^{\infty} 2^{i+1} k_{i}(B) \leq 2^{d}\left(k(B)-l\left(b_{z}\right)\right) .
\end{gathered}
$$

In particular, Brauer's $k(B)$-conjecture holds if $k(B)-l\left(b_{z}\right) \leq 3$.

Proof. By [Feit 1982, Lemma V.9.3], the numbers $m_{\chi \chi}^{\prime}$ for $\chi \in \operatorname{Irr}(B)$ are still real, positive algebraic integers. As in Theorem 2.2, we may assume $|\langle z\rangle|=2^{n} \geq 4$. Let us write

$$
m_{\chi \chi}^{\prime}=\sum_{j=0}^{2^{n-1}-1} a_{j}(\chi) \zeta^{j}
$$

with $\chi \in \operatorname{Irr}_{0}(B), \zeta:=e^{2 \pi i / 2^{n}}$ and $a_{j}(\chi) \in \mathbb{Z}$ for $j=0, \ldots, 2^{n-1}-1$. The Galois group still acts on $M_{z}^{\prime}$. Also the equation $\left(M_{z}^{\prime}\right)^{2}=M_{z}^{\prime} \bar{M}_{z}^{\prime}$ ' $=2^{d} M_{z}^{\prime}$ remains true. For $\tau \in \operatorname{Irr}(B)$, we have $v\left(m_{\chi \tau}^{\prime}\right)=v\left(2^{d}-m_{\chi \tau}^{z}\right)=v\left(m_{\chi \tau}^{z}\right)=h(\tau)$. Hence, in case $a_{0}(\chi) \leq \alpha$ we can carry over the arguments in Theorem 2.2.

Now assume that $a_{0}(\chi)>\alpha$ for all characters $\chi \in \operatorname{Irr}_{0}(B)$. Here too the proof works much as in Theorem 2.2. In fact, for a character $\psi \in \operatorname{Irr}(B)$ of positive height we have $v\left(m_{\psi \psi}^{\prime}\right)=v\left(2^{d}-m_{\psi \psi}^{z}\right) \geq \min \left\{v\left(2^{d}\right), v\left(m_{\psi \psi}^{z}\right)\right\}>h(\psi)$ by [Feit 1982, Theorem V.9.4]. Moreover, $\operatorname{tr} M_{z}^{\prime}=2^{d}(k(B)-l(B))$. The claim follows.

It should be pointed out that usually $k(B)-l(B)=k(B)-l\left(b_{1}\right) \leq k(B)-l\left(b_{z}\right)$ for a major subsection $\left(z, b_{z}\right)$ (this holds for example if $z$ lies in the center of the fusion system of $B$; see [Külshammer and Okuyama $\sim 2000])$. However, this is not true in general as we see in [Külshammer and Sambale 2013, Proposition 2.1(vii)]. Another problem is that $k(B)-l\left(b_{z}\right)$ for $z \neq 1$ is not locally determined (in contrast to $k(B)-l(B)$ ). By combining with Proposition 2.3, we can replace Proposition 2.5(2) by

$$
(\alpha+2) k_{0}(B)+\sum_{i=1}^{\infty} 2^{i+1} k_{i}(B) \leq 2^{d} \min \left\{l\left(b_{z}\right), k(B)-l\left(b_{z}\right)\right\} .
$$

\section{Bicyclic defect groups}

As mentioned in the introduction, we consider in this section blocks with defect groups coming from [Sambale 2012b, Theorem 4.19]. A key feature of the groups in the next three theorems is that all their irreducible characters have degree 1 or 2. We also remark that Olsson's conjecture was verified for all blocks with bicyclic defect groups in [Sambale 2012b]. 
Theorem 3.1. Let B be a nonnilpotent 2-block of a finite group with defect group

$$
D \cong\left\langle v, x, a \mid v^{2^{n}}=x^{2}=a^{2^{m}}=1,{ }^{x} v={ }^{a} v=v^{-1},{ }^{a} x=v x\right\rangle \cong D_{2^{n+1}} \rtimes C_{2^{m}}
$$

for some $n, m \geq 2$. Then $k(B)=2^{m-1}\left(2^{n}+3\right), k_{0}(B)=2^{m+1}, k_{1}(B)=2^{m-1}\left(2^{n}-1\right)$ and $l(B)=2$. In particular, Brauer's $k(B)$-conjecture and Alperin's weight conjecture are satisfied.

Proof. Let $\mathscr{F}$ be the fusion system of $B$, and let $z:=v^{2^{n-1}}$. Then by [Sambale

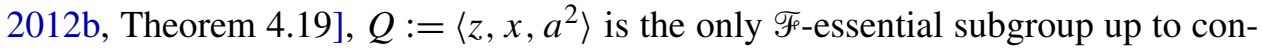
jugation. In order to calculate $k(B)$, we use Brauer's formula [Nagao and Tsushima 1989, Theorem 5.9.4]. We will see that it is not necessary to obtain a complete set of representatives for the $\mathscr{F}$-conjugacy classes. Since $\langle v, a x\rangle$ is an abelian maximal subgroup of $D$, all characters in $\operatorname{Irr}(D)$ have degree 1 or 2 . In particular, $k(D):=|\operatorname{Irr}(D)|=\left|D / D^{\prime}\right|+\left(|D|-\left|D / D^{\prime}\right|\right) / 4=2^{m-1}\left(2^{n}+3\right)$. Now we have to count how many conjugacy classes of $D$ are fused under $\operatorname{Aut}_{\mathscr{F}}(Q)$. According to [Sambale 2012b, Theorem 4.19], there are two possibilities $\mathrm{C}_{Q}\left(\operatorname{Aut}_{\mathscr{F}}(Q)\right)=$ $\mathrm{Z}(\mathscr{F}) \in\left\{\left\langle a^{2}\right\rangle,\left\langle a^{2} z\right\rangle\right\}$. In the first case, the elements of the form $x a^{2 j}$ are conjugate to corresponding elements $z a^{2 j}$ under $\operatorname{Aut}_{\mathscr{F}}(Q)$. In the second case, a similar statement is true for $a^{2 j}$. Observe that the elements $x a^{2^{j}}$ and $x z a^{2 j}$ are already conjugate in $D$. Since $\left\langle a^{2}, z\right\rangle \subseteq \mathrm{Z}(D)$, no more fusion can occur. Hence, the number

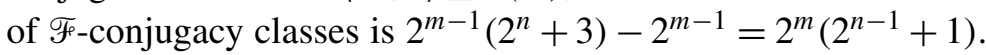

Now we have to determine at least some of the numbers $l\left(b_{u}\right)$ where $u \in D$. The groups $\bar{D}_{1}:=D /\left\langle a^{2}\right\rangle$ and $\bar{D}_{2}:=D /\left\langle a^{2} z\right\rangle$ have commutator subgroups $D^{\prime}\left\langle a^{2}\right\rangle /\left\langle a^{2}\right\rangle$ and $D^{\prime}\left\langle a^{2} z\right\rangle /\left\langle a^{2} z\right\rangle$ of index 4 , respectively. Hence, $\bar{D}_{1}$ and $\bar{D}_{2}$ have maximal class. The blocks $b_{a^{2}}$ and $b_{a^{2} z}$ dominate blocks $\overline{b_{a^{2}}}$ and $\overline{b_{a^{2}} z}$, respectively, with defect group $\bar{D}_{1}$. Let $\mathscr{F}_{1}$ and $\mathscr{F}_{2}$ be the fusion systems of $\overline{b_{a^{2}}}$ and $\overline{b_{a^{2}} z}$, respectively. Then in case $\mathrm{Z}(\mathscr{F})=\left\langle a^{2}\right\rangle$ or $\mathrm{Z}(\mathscr{F})=\left\langle a^{2} z\right\rangle, \bar{Q}$ is the only $\mathscr{F}_{1}$-essential or $\mathscr{F}_{2}$-essential subgroup of $\bar{D}_{1}$ or $\bar{D}_{2}$, respectively, up to conjugation. Thus, [Brauer 1974; Olsson 1975] imply $l\left(b_{a^{2}}\right)=l\left(\overline{b_{a^{2}}}\right)=2$ and $l\left(b_{a^{2} z}\right)=l\left(\overline{b_{a^{2} z}}\right)=2$. The same holds for all odd powers of $a^{2}$ and $a^{2} z$. Next we consider the elements $u:=a^{2^{j}}$ for $2 \leq j \leq m-1$. It can be seen that the isomorphism type of $D /\langle u\rangle$ is the same as for $D$ except that we have to replace $m$ by $j$. Also the essential subgroup $Q$ carries over to the block $\overline{b_{u}}$. Hence, induction on $m$ gives $l\left(b_{u}\right)=2$ as well. For all other nontrivial subsections $\left(u, b_{u}\right)$, we only know $l\left(b_{u}\right) \geq 1$. Finally, $l(B) \geq 2$ since $B$ is centrally controlled, by Theorem 1.1 in [Külshammer and Okuyama 2000]. Applying Brauer's formula gives

$$
k(B) \geq 2^{m}+2^{m}\left(2^{n-1}+1\right)-2^{m-1}=2^{m-1}\left(2^{n}+3\right)=k(D) .
$$

We already know from [Sambale 2012b, Theorem 5.3] that Olsson's conjecture holds for $B$, i.e., $k_{0}(B) \leq\left|D: D^{\prime}\right|=2^{m+1}$. Now we apply [Robinson 1991, Theorem 3.4] 
to the subsection $\left(z, b_{z}\right)$, which gives

$$
|D|=2^{m+1}+2^{m+1}\left(2^{n}-1\right) \leq k_{0}(B)+4\left(k(B)-k_{0}(B)\right) \leq \sum_{i=0}^{\infty} 2^{2 i} k_{i}(B) \leq|D| .
$$

This implies $k(B)=k(D)=2^{m-1}\left(2^{n}+3\right), k_{0}(B)=2^{m+1}, k_{1}(B)=2^{m-1}\left(2^{n}-1\right)$ and $l(B)=2$. Brauer's $k(B)$-conjecture follows immediately. In order to prove Alerin's weight conjecture (see [Kessar 2007, Proposition 5.4]), it suffices to show that $Q$ and $D$ are the only $\mathscr{F}$-radical, $\mathscr{F}$-centric subgroups of $D$. Thus, assume by way of contradiction that $Q_{1}$ is another $\mathscr{F}$-radical, $\mathscr{F}$-centric subgroup. Since $Q_{1}$ is $\mathscr{F}_{F}$-centric, it cannot lie inside $Q$. Moreover, $\operatorname{Out}_{\mathscr{F}}\left(Q_{1}\right)$ must provide a morphism of odd order because $Q_{1}<D$. However, by Alperin's fusion theorem $\mathscr{F}$ is generated by $\operatorname{Aut}_{\mathscr{F}}(Q)$ and $\operatorname{Aut}_{\mathscr{F}}(D)$. This gives the desired contradiction.

We add some remarks. First, the direct products of similar type $D_{2^{n+1}} \times C_{2^{m}}$ were already handled in [Sambale 2012a]. Also if $n=1$, we obtain the minimal nonabelian group $C_{2}^{2} \rtimes C_{2^{m}}$ for which the block invariants are also known by [Sambale 2011a]. Moreover, it is an easy exercise to check that various other conjectures (for example [Eaton and Moretó 2013; Eaton 2003; Malle and Navarro 2006]) are also true in the situation of Theorem 3.1. We will not go into the details here.

The next theorem concerns defect groups that have a similar structure as the central products $Q_{2^{n+1}} * C_{2^{m}}$ discussed in [Sambale 2013a]. Also, this result is needed for the induction step in the theorem after that.

Theorem 3.2. Let $B$ be a nonnilpotent 2-block of a finite group with defect group

$$
\begin{aligned}
D \cong\langle v, x, a| v^{2^{n}}=1, a^{2^{m}}=x^{2}=v^{2^{n-1}},{ }^{x} v={ }^{a} v=v^{-1} & \left.,{ }^{a} x=v x\right\rangle \\
& \cong Q_{2^{n+1}} . C_{2^{m}} \cong D_{2^{n+1}} . C_{2^{m}}
\end{aligned}
$$

for some $n, m \geq 2$ and $m \neq n$. Then $k(B)=2^{m+1}\left(2^{n-2}+1\right), k_{0}(B)=2^{m+1}$, $k_{1}(B)=2^{m-1}\left(2^{n}-1\right), k_{n}(B)=2^{m-1}$ and $l(B)=2$. In particular, Brauer's $k(B)$-conjecture and Alperin's weight conjecture are satisfied.

Proof. First observe that the proof of [Sambale 2012b, Theorem 4.20] shows that in fact

$$
D \cong\left\langle v, x, a \mid v^{2^{n}}=x^{2}=1, a^{2^{m}}=v^{2^{n-1}},{ }^{x} v={ }^{a} v=v^{-1},{ }^{a} x=v x\right\rangle \cong D_{2^{n+1}} . C_{2^{m}} .
$$

Let $\mathscr{F}$ be the fusion system of $B$, and let $y:=v^{2^{n-2}}$ and $z:=x^{2}$. Then by [Sambale 2012b, Theorem 4.19], $Q:=\left\langle x, y, a^{2}\right\rangle \cong Q_{8} * C_{2^{m}}$ is the only $\mathscr{F}_{\text {-essential subgroup }}$ up to conjugation (since $n \neq m, D$ is not a wreath product). Again we use Brauer's formula [Nagao and Tsushima 1989, Theorem 5.9.4] to get a lower bound for $k(B)$. The same argumentation as in Theorem 3.1 shows that $D$ has $2^{m-1}\left(2^{n}+3\right)$ conjugacy classes, and we need to know which of them are fused in $Q$. It is easy to 
see that $x a^{2 j}$ is conjugate to $y a^{2 j}$ under $\operatorname{Aut}_{\mathscr{F}}(Q)$ for $j \in \mathbb{Z}$. Observe that $x a^{2 j}$ is already conjugate to $x y a^{2^{j}}$ and $x^{-1} a^{2^{j}}=x a^{2 j+2^{m}}$ in $D$. Since $\mathrm{Z}(\mathscr{F})=\left\langle a^{2}\right\rangle$, this is the only fusion that occurs. Hence, the number of $\mathscr{F}$-conjugacy classes is again $2^{m}\left(2^{n-1}+1\right)$.

Again $D /\left\langle a^{2}\right\rangle$ has maximal class and $l\left(b_{a^{2}}\right)=2$ by [Brauer 1974; Olsson 1975]. The same is true for the odd powers of $a^{2}$. Now let $u:=a^{2^{j}}$ for some $2 \leq j \leq m$. Then it turns out that $D /\langle u\rangle$ is isomorphic to the group $D_{2^{n}} \rtimes C_{2^{j}}$ as in Theorem 3.1. So we obtain $l\left(b_{u}\right)=2$ as well. For the other nontrivial subsections $\left(u, b_{u}\right)$, we have at least $l\left(b_{u}\right) \geq 1$. Finally, $l(B) \geq 2$ since $B$ is centrally controlled (see [Külshammer and Okuyama $\sim 2000$, Theorem 1.1]). Therefore,

$$
k(B) \geq 2^{m+1}+2^{m}\left(2^{n-1}+1\right)-2^{m}=2^{m+1}\left(2^{n-2}+1\right) .
$$

Also, $k_{0}(B) \leq 2^{m+1}$ by [Sambale 2012b, Theorem 5.3]. However, in this situation we cannot apply [Robinson 1991]. So we use [Héthelyi et al. 2013, Theorem 2.4] for the major subsection $\left(a^{2}, b_{a^{2}}\right)$. Let us determine the isomorphism type of $\bar{D}:=D /\left\langle a^{2}\right\rangle$ precisely. Since $(a x)^{2}=a x a x=v x^{2} a^{2} \equiv v\left(\bmod \left\langle a^{2}\right\rangle\right)$, ax generates a cyclic maximal subgroup $\bar{D}$. Since ${ }^{a}(a x)=a v x=a x v^{-1} \equiv(a x)^{-1}\left(\bmod \left\langle a^{2}\right\rangle\right)$, $\bar{D} \cong D_{2^{n+1}}$. Hence, the Cartan matrix of $b_{a^{2}}$ is given by

$$
2^{m}\left(\begin{array}{cc}
2^{n-1}+1 & 2 \\
2 & 4
\end{array}\right)
$$

up to basic sets (see [Erdmann 1990]). This gives $k(B) \leq 2^{m}\left(2^{n-1}+3\right)$, which is not quite what we wanted. However, the restriction on $k_{0}(B)$ will show that this maximal value for $k(B)$ cannot be reached. For this, we use the same method as in [Sambale 2013a]; i.e., we analyze the generalized decomposition numbers $d_{\chi \varphi_{i}}^{u}$ for $u:=a^{2}$ and $\operatorname{IBr}\left(b_{u}\right)=\left\{\varphi_{1}, \varphi_{2}\right\}$. Since the argument is quite similar except that $n$ has a slightly different meaning, we only present some key observations here. As in [Sambale 2013a], we write

$$
d_{\chi \varphi_{i}}^{u}=\sum_{j=0}^{2^{m-1}-1} a_{j}^{i}(\chi) \zeta^{j}
$$

where $\zeta:=e^{2 \pi i / 2^{m}}$. It follows that

$$
\left(a_{i}^{1}, a_{j}^{1}\right)=\left(2^{n}+2\right) \delta_{i j}, \quad\left(a_{i}^{1}, a_{j}^{2}\right)=4 \delta_{i j} \quad \text { and } \quad\left(a_{i}^{2}, a_{j}^{2}\right)=8 \delta_{i j} .
$$

Moreover, $h(\chi)=0$ if and only if $\sum_{j=0}^{2^{m-1}-1} a_{j}^{2}(\chi) \equiv 1(\bmod 2)$. This gives three essentially different possibilities for $a_{j}^{1}$ and $a_{j}^{2}$ as in [Sambale 2013a]. Let the 
numbers $\alpha, \beta, \gamma$ and $\delta$ be defined as there. Then

$$
\begin{aligned}
\gamma & =2^{m-1}-\alpha-\beta, \\
k(B) & \leq\left(2^{n}+6\right) \alpha+\left(2^{n}+4\right) \beta+\left(2^{n}+2\right) \gamma-\delta / 2 \\
& =2^{m+n-1}+6 \alpha+4 \beta+2 \gamma-\delta / 2 \\
& =2^{m+n-1}+2^{m}+4 \alpha+2 \beta-\delta / 2, \\
8 \alpha+4 \beta-\delta & \leq k_{0}(B) \leq 2^{m+1} .
\end{aligned}
$$

This shows $k(B) \leq 2^{m+n-1}+2^{m+1}=2^{m+1}\left(2^{n-2}+1\right)$. Together with (1), we have $k(B)=2^{m+1}\left(2^{n-2}+1\right)$ and $l(B)=2$. The inequalities above also show $k_{0}(B)=2^{m+1}$. Now we can carry over the further discussion in [Sambale 2013a] word by word. In particular, we get $\delta=0$,

$$
\begin{aligned}
k_{1}(B) & =\left(2^{n}-2\right) \alpha+\left(2^{n}-1\right) \beta+2^{n} \gamma=2^{n+m-1}-2 \alpha-\beta \\
& =2^{n+m-1}-2^{m-1}=2^{m-1}\left(2^{n}-1\right)
\end{aligned}
$$

and finally $k_{n}(B)=2^{m-1}$. The conjectures follow as usual.

Now we can also handle defect groups of type $Q_{2^{n+1}} \rtimes C_{2^{m}}$. It is interesting to see that we get the same number of characters although the groups are nonisomorphic as shown in [Sambale 2012b].

Theorem 3.3. Let $B$ be a nonnilpotent 2-block of a finite group with defect group $D \cong\left\langle v, x, a \mid v^{2^{n}}=a^{2^{m}}=1, x^{2}=v^{2^{n-1}},{ }^{x} v={ }^{a} v=v^{-1},{ }^{a} x=v x\right\rangle \cong Q_{2^{n+1}} \rtimes C_{2^{m}}$ for some $n, m \geq 2$. Then $k(B)=2^{m+1}\left(2^{n-2}+1\right), k_{0}(B)=2^{m+1}, k_{1}(B)=$ $2^{m-1}\left(2^{n}-1\right), k_{n}(B)=2^{m-1}$ and $l(B)=2$. In particular, Brauer's $k(B)$-conjecture and Alperin's weight conjecture are satisfied.

Proof. Let $\mathscr{F}$ be the fusion system of $B$, and let $y:=v^{2^{n-2}}$ and $z:=x^{2}$. Then by [Sambale 2012b, Theorem 4.19], $Q:=\left\langle x, y, a^{2}\right\rangle \cong Q_{2^{n+1}} \times C_{2^{m-1}}$ is the only $\mathscr{F}$-essential subgroup up to conjugation. Again we use Brauer's formula [Nagao and Tsushima 1989, Theorem 5.9.4] to get a lower bound for $k(B)$.

The same argument as in Theorem 3.1 shows that $D$ has $2^{m-1}\left(2^{n}+3\right)$ conjugacy classes and we need to know which of them are fused in $Q$. It is easy to see that $x a^{2 j}$ is conjugate to $y a^{2 j}$ under $\operatorname{Aut}_{\mathscr{F}}(Q)$ for $j \in \mathbb{Z}$. Since $\mathrm{Z}(\mathscr{F})=\left\langle z, a^{2}\right\rangle$, this is the only fusion that occurs. Hence, the number of $\mathscr{F}$-conjugacy classes is again $2^{m}\left(2^{n-1}+1\right)$. In case $n=2$, the group $D /\langle z\rangle \cong C_{2}^{2} \rtimes C_{2^{m}}$ is minimal nonabelian, and we get $l\left(b_{z}\right)=2$ from [Sambale 2011a]. Otherwise, $D /\langle z\rangle$ is isomorphic to one of the groups in Theorem 3.1. Hence, again $l\left(b_{z}\right)=2$. As usual, the groups $D /\left\langle a^{2}\right\rangle$ and $D /\left\langle a^{2} z\right\rangle$ have maximal class and it follows that $l\left(b_{a^{2}}\right)=l\left(b_{a^{2} z}\right)=2$. The same holds for all odd powers of $a^{2}$ and $a^{2} z$. For $2 \leq j \leq m-1$, the group $D /\langle u\rangle$ 
with $u:=a^{2^{j}}$ has the same isomorphism type as $D$ where $m$ has to be replaced by $j$. So induction on $m$ shows $l\left(b_{u}\right)=2$. It remains to deal with $u:=a^{2^{j}} z$. Here $D /\langle u\rangle \cong Q_{2^{n+1}} . C_{2^{j}}$ is exactly the group from Theorem 3.2. Thus, for $j \neq n$ we have again $l\left(b_{u}\right)=2$. In case $j=n, D /\langle u\rangle \cong C_{2^{n}} C_{2}$. Then [Külshammer 1980, (7.G)] gives $l\left(b_{u}\right)=2$ as well. Now Brauer's formula reveals

$$
k(B) \geq 2^{m+1}+2^{m}\left(2^{n-1}+1\right)-2^{m}=2^{m+1}\left(2^{n-2}+1\right) .
$$

For the opposite inequality, we apply [Héthelyi et al. 2013, Theorem 2.4] to the major subsection $\left(u, b_{u}\right)$ where $u:=a^{2} z$. A similar calculation as in Theorem 3.2 shows that $D /\langle u\rangle \cong Q_{2^{n+2}}$. Hence, the Cartan matrix of $b_{u}$ is given by

$$
2^{m}\left(\begin{array}{cc}
2^{n-1}+1 & 2 \\
2 & 4
\end{array}\right)
$$

up to basic sets (see [Erdmann 1990]). This is the same matrix as in Theorem 3.2, but the following discussion is slightly different because $a^{2}$ has only order $2^{m-1}$ here. So we copy the proof of the main theorem in [Sambale 2013b]. In fact, we just have to replace $m$ with $m+1$ and $n$ with $n-2$ in order to use this proof word for word. The claim follows.

We describe the structure of these group extensions in a more generic way.

Proposition 3.4. Let $D$ be an extension of the cyclic group $\langle a\rangle \cong C_{2^{n}}$ by a group $M$ that has maximal class or is the four-group. Suppose that the corresponding coupling $\omega:\langle a\rangle \rightarrow \operatorname{Out}(M)$ satisfies the following: if $\omega \neq 0$, then the coset $\omega(a)$ of $\operatorname{Inn}(M)$ contains an involution that acts nontrivially on $M / \Phi(M)$. Moreover, assume that $D ¥ C_{2^{m}} ح C_{2}$ for all $m \geq 3$. Then the invariants for every block of a finite group with defect group D are known.

Proof. Assume first that $M \cong C_{2}^{2}$. Then in case $\omega=0$, we get the groups $C_{2^{n}} \times C_{2}^{2}$ and $C_{2^{n+1}} \times C_{2}$ for which the block invariants can be calculated by [Usami 1988; Kessar et al. 2012]. So let $\omega \neq 0$. If $D$ is nonsplit, it must contain a cyclic maximal subgroup. In particular, $D$ is metacyclic and the block invariants are known. If the extension splits, we obtain the minimal nonabelian group $C_{2}^{2} \rtimes C_{2^{n}}$. Here the block invariants are known by [Sambale 2011a].

Hence, let $M$ be a 2-group of maximal class. Then $|\mathrm{Z}(M)|=2$. Thus, for $\omega=0$ we obtain precisely two extensions for every group $M$. All these cases were handled in [Sambale 2012a; 2013b; 2013a]. Let us now consider the case $\omega \neq 0$. Since the three maximal subgroups of a semidihedral group are pairwise nonisomorphic, $M$ must be a dihedral or quaternion group. Write $M=\left\langle v, x \mid v^{2^{m}}=1, x^{2} \in\left\langle v^{2^{m-1}}\right\rangle,{ }^{x} v=v^{-1}\right\rangle$. Let $\alpha \in \operatorname{Aut}(M)$ be an involution that acts nontrivially on $M / \Phi(M)$. Then there is an odd integer $i$ such that ${ }^{\alpha} x=v^{i} x$. Since $\alpha^{2}=1$, it follows that ${ }^{\alpha} v=v^{-1}$. Hence, the $\operatorname{coset} \alpha \operatorname{Inn}(M) \in \operatorname{Out}(M)$ is determined uniquely. Hence, $\omega$ is unique. 
So we get four group extensions for every pair $(n, m)$. Two of them are isomorphic, and all cases are covered in Theorems 3.1, 3.2 and 3.3 (and [Külshammer 1980] for $C_{4} \succ C_{2}$ ).

\section{More examples}

Since almost all block invariants for 2-blocks of defect 4 are known (see [Külshammer and Sambale 2013]), it is natural to look at 2-blocks of defect 5. Here for the abelian defect group $C_{4} \times C_{2}^{3}$, the invariants are not known so far. We handle more general abelian defect groups in the next theorem. This result relies on the classification of the finite simple groups. We denote the inertial index of $B$ by $e(B)$.

Theorem 4.1. Let $B$ be a block of a finite group $G$ with defect group $C_{2^{n}} \times C_{2}^{3}$ for some $n \geq 2$. Then we have $k(B)=k_{0}(B)=|D|=2^{n+3}$ and one of the following holds:

(i) $e(B)=l(B)=1$.

(ii) $e(B)=l(B)=3$.

(iii) $e(B)=l(B)=7$.

(iv) $e(B)=21$ and $l(B)=5$.

Proof. Let $D=C_{2^{n}} \times C_{2}^{3}$. Since $\operatorname{Aut}(D)$ acts faithfully on $\Omega(D) / \Phi(D) \cong C_{2}^{3}$, we have $e(B) \in\{1,3,7,21\}$. In case $e(B)=1$, the block is nilpotent and the result is clear. Now we consider the remaining cases.

Case 1: $e(B)=3$. Then there are $2^{n+2}$ subsections $\left(u, b_{u}\right)$ up to conjugation and $2^{n+1}$ of them satisfy $l\left(b_{u}\right)=1$. For the other $2^{n+1}$ subsections, [Watanabe 1991, Theorem 1] implies $l\left(b_{u}\right)=3$. This gives $k(B)=2^{n+3}=|D|$. The height-0 conjecture follows from [Kessar and Malle 2013, Theorem 1.1].

Case 2: $e(B)=7$. Here we have $2^{n+1}$ subsections $\left(u, b_{u}\right)$ up to conjugation, where $2^{n}$ of them satisfy $l\left(b_{u}\right)=1$. For the other $2^{n}$ subsections, we use [Watanabe 1991, Theorem 1] in connection with [Kessar et al. 2012, Theorem 1.1] (instead of [Kessar et al. 2012], we could also use [Kessar and Malle 2013], which we need anyway). This gives $l\left(b_{u}\right)=7$ for these subsections. It follows that $k(B)=|D|$ and $k(B)=k_{0}(B)$ by [Kessar and Malle 2013, Theorem 1.1].

Case 3: $e(B)=21$. Here we have again $2^{n+1}$ subsections $\left(u, b_{u}\right)$ up to conjugation. But this time $2^{n}$ subsections satisfy $l\left(b_{u}\right)=3$ and the other $2^{n}$ subsections satisfy $l\left(b_{u}\right)=5$ by [Watanabe 1991; Kessar et al. 2012]. The result follows as before.

Next we study another group of order 32 with an easy structure. For this, let $\operatorname{MNA}(r, s)$ be the minimal nonabelian group given by

$$
\left\langle x, y \mid x^{2^{r}}=y^{2^{s}}=[x, y]^{2}=[x, x, y]=[y, x, y]=1\right\rangle
$$


for some $r \geq s \geq 1$ (see [Rédei 1947]). For the notion of a constrained fusion system, we refer to [Oliver and Ventura 2009, Definition 2.3].

Proposition 4.2. Let $B$ be a nonnilpotent block of a finite group with defect group $D \cong \operatorname{MNA}(2,1) \times C_{2}$. Then $k(B)=20, k_{0}(B)=16, k_{1}(B)=4$ and $l(B)=2$. In particular, Olsson's conjecture and Alperin's weight conjecture hold for $B$.

Proof. Let $\mathscr{F}$ be the fusion system of $B$. Since $|D: Z(D)|=4$, every $\mathscr{F}$-essential subgroup is maximal, and there are three candidates for these groups. Let $\mathrm{Z}(D)<M<D$ such that $M \cong C_{4} \times C_{2}^{2}$. Then $\operatorname{Aut}_{\mathscr{F}}(M)$ must act nontrivially on $\Omega(M) / \Phi(M)$. However, it can be seen that $\mathrm{N}_{D}(M)$ acts trivially on $\Omega(M) / \Phi(M)$. In particular, $M$ is not $\mathscr{F}$-radical. Hence, there is only one $\mathscr{F}$-essential subgroup $Q \cong C_{2}^{4}$ (up to conjugation). Since $Q \unlhd D, \mathscr{F}$ is constrained and thus uniquely determined by $\operatorname{Out}_{\mathscr{F}}(Q)$ (see [Linckelmann 2007, Theorem 4.6]). By [Sambale 2012b, Lemma 3.11], we have some possibilities for $\operatorname{Out}_{\mathscr{F}}(Q)$. However, a GAP calculation shows that only $\operatorname{Out}_{\mathscr{F}}(Q) \cong S_{3}$ is realizable. Then $\mathscr{F}_{F}$ is the fusion system on the group SmallGroup $(96,194) \cong\left(A_{4} \rtimes C_{4}\right) \times C_{2}$. In particular, there are exactly $16 \mathscr{F}_{\text {- }}$ conjugacy classes on $D$. Moreover, $\mathrm{Z}(\mathscr{F}) \cong C_{2}^{2}$, and for $1 \neq z \in \mathrm{Z}(\mathscr{F})$, we have $D /\langle z\rangle \in\left\{\operatorname{MNA}(2,1), D_{8} \times C_{2}\right\}$. Hence, we get $l\left(b_{z}\right)=2$ as usual. For all other nontrivial subsections $\left(u, b_{u}\right)$, we have $l\left(b_{u}\right) \geq 1$. Since $B$ is centrally controlled, [Külshammer and Okuyama $\sim 2000$, Theorem 1.1] implies $l(B) \geq 2$. Brauer's formula for $k(B)$ gives $k(B) \geq 20$. If $x \in D$ has order 4 , then $C_{D}(x) /\langle x\rangle$ has order 4 . Hence, Olsson's conjecture follows from [Héthelyi et al. 2013, Theorem 2.5]; i.e., $k_{0}(B) \leq\left|D: D^{\prime}\right|=16$. For an element $z \in \mathrm{Z}(D) \backslash \mathrm{Z}(\mathscr{F})$, the block $b_{z}$ is nilpotent. Thus, [Robinson 1991, Theorem 3.4] implies

$$
|D|=32 \leq k_{0}(B)+4\left(k(B)-k_{0}(B)\right) \leq \sum_{i=0}^{\infty} 2^{2 i} k_{i}(B) \leq|D| .
$$

The claim follows as usual.

In the classification of the simple groups of 2-rank 2, the sole exception PSU $(3,4)$ shows up (see [Alperin et al. 1973]). This group has a Suzuki Sylow 2-subgroup $P$ of order 64 (see [Craven and Glesser 2012, Definition 1.4]). The group $P$ also occurs in the classification of the center-free fusion systems on 2-groups of 2-rank 2 (see [Craven and Glesser 2012]). It can also be described as the smallest 2-group with exactly three involutions and an automorphism of order 5. This answers a question raised in [Berkovich and Janko 2008, Exercise 82.3]. In fact, $P$ admits an automorphism of order 15 . Moreover, $\mathrm{Z}(P)=\Phi(P)=P^{\prime}=\Omega(P) \cong C_{2}^{2}$, so $P$ is special (see [Gorenstein 1968, p. 183]).

Using this as a motivation, it seems worthwhile to obtain the invariants of blocks with defect group $P$ (this will be done in an upcoming diploma thesis). Doing so, 
we need to handle the extraspecial group $P /\langle z\rangle \cong D_{8} * Q_{8}$ for $1 \neq z \in \mathrm{Z}(P)$ for the induction step.

Proposition 4.3. Let $B$ be a block of a finite group $G$ with defect group $D_{8} * Q_{8}$ and inertial index 5. Then $l(B)=5, k(B)=13, k_{0}(B)=8$ and $k_{2}(B)=5$. Moreover, the Cartan matrix of $B$ is given by

$$
2\left(\begin{array}{lllll}
2 & 1 & 1 & 1 & 1 \\
1 & 2 & 1 & 1 & 1 \\
1 & 1 & 2 & 1 & 1 \\
1 & 1 & 1 & 2 & 1 \\
1 & 1 & 1 & 1 & 4
\end{array}\right)
$$

up to basic sets.

Proof. Let $D=D_{8} * Q_{8}$, and let $\mathscr{F}$ be the fusion system of $B$. By [Stancu 2006, Theorem 5.3], $\mathscr{F}$ is controlled by $\operatorname{Aut}_{\mathscr{F}}(D)$. Let $\mathrm{Z}(D)=\langle z\rangle$. As usual, we denote the subsections by $\left(u, b_{u}\right)$. Then $b_{z}$ covers a block $\bar{b}_{z}$ with elementary abelian defect group of order 16. It follows from [Külshammer and Sambale 2013, Proposition 2.1] that $5=e(B)=e\left(b_{z}\right)=e\left(\bar{b}_{z}\right)=l\left(\bar{b}_{z}\right)=l\left(b_{z}\right)$. Moreover, $B$ is centrally controlled; in particular, [Külshammer and Okuyama $\sim 2000$, Theorem 1.1] implies $l(B) \geq 5$.

There are three nonmajor subsections $\left(u_{1}, b_{1}\right),\left(u_{2}, b_{2}\right)$ and $\left(u_{3}, b_{3}\right)$. Since $\left|D^{\prime}\right|=2$, every conjugacy class in $D$ has at most two elements. In particular, $\left|\mathrm{C}_{D}\left(u_{i}\right)\right|=16$ for $i=1,2,3$. By [Héthelyi et al. 2013, Proposition 5.1], we have $l\left(b_{i}\right)=1$ for $i=1,2,3$. Now let us look at the major subsection $\left(z, b_{z}\right)$. By the proof of [Sambale 2011c, Proposition 1], the Cartan matrix of $b_{z}$ is given by

$$
2\left(\begin{array}{lllll}
4 & 3 & 3 & 3 & 3 \\
3 & 4 & 3 & 3 & 3 \\
3 & 3 & 4 & 3 & 3 \\
3 & 3 & 3 & 4 & 3 \\
3 & 3 & 3 & 3 & 4
\end{array}\right)
$$

up to basic sets. If we change the basic set, we get the following matrix with smaller entries:

$$
C_{z}:=2\left(\begin{array}{lllll}
2 & 1 & 1 & 1 & 1 \\
1 & 2 & 1 & 1 & 1 \\
1 & 1 & 2 & 1 & 1 \\
1 & 1 & 1 & 2 & 1 \\
1 & 1 & 1 & 1 & 4
\end{array}\right)
$$

Now we consider the matrix $D_{z}:=\left(d_{i j}^{z}\right)$. Since $z$ has order $2, D_{z}$ is an integral matrix such that $D_{z}^{\mathrm{T}} D_{z}=C$. Since all columns of $D_{z}$ are orthogonal to the columns of ordinary decomposition numbers, we see that the first four columns consist of 
exactly four entries \pm 1 each. By way of contradiction, assume that the first two columns of $D_{z}$ have the form

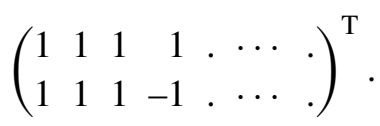

Then there is at least one column of ordinary decomposition numbers that is not orthogonal to the difference of these two columns of $D_{z}$. This contradiction shows that $D_{z}$ has the form

$$
D_{z}=\left(\begin{array}{ccccccccccccc}
1 & 1 & 1 & 1 & . & . & . & . & . & . & . & \cdots & . \\
1 & 1 & . & . & 1 & 1 & . & . & . & . & . & \cdots & . \\
1 & 1 & . & . & . & . & 1 & 1 & . & . & . & \cdots & . \\
1 & 1 & . & . & . & . & . & . & 1 & 1 & . & \cdots & . \\
* & * & * & * & * & * & * & * & * & * & * & \cdots & *
\end{array}\right)^{\mathrm{T}}
$$

up to signs and permutations. It holds that $k(B)=l(B)+l\left(b_{z}\right)+l\left(b_{1}\right)+l\left(b_{2}\right)+$ $l\left(b_{3}\right) \geq 13$. Hence, for the last column of $D_{z}$ we have essentially the following possibilities:

$$
\begin{aligned}
& \left(\begin{array}{lllllllllllll}
1 & 1 & \ldots & \ldots & \ldots & \ldots & 1 & 1 & 1 & 1 & 1 & 1
\end{array}\right)^{\mathrm{T}} \text {, } \\
& (1.1 .1 .1 .1 \cdot 111)^{\mathrm{T}} \text {, } \\
& \left(\begin{array}{llllllllllll}
1 & 1 & 1 & -1 & \ldots & \ldots & 1 & 1 & 1 & 1
\end{array}\right)^{\mathrm{T}} \text {, }
\end{aligned}
$$

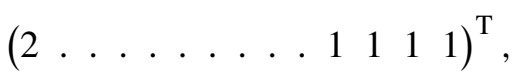

$$
\begin{aligned}
& \left(\begin{array}{llllllllll}
1 & 1 & \ldots & \ldots & 2 & 1 & 1
\end{array}\right)^{\mathrm{T}} \text {. }
\end{aligned}
$$

This already implies $k(B) \in\{13,14,16\}$. In order to investigate the heights of the irreducible characters, we consider the matrix $M^{z}=\left(m_{i j}^{z}\right)=32 D_{z} C_{z}^{-1} D_{z}^{\mathrm{T}}$ of contributions. We have

$$
32 C_{z}^{-1}=\left(\begin{array}{rrrrr}
13 & -3 & -3 & -3 & -1 \\
-3 & 13 & -3 & -3 & -1 \\
-3 & -3 & 13 & -3 & -1 \\
-3 & -3 & -3 & 13 & -1 \\
-1 & -1 & -1 & -1 & 5
\end{array}\right)
$$

By [Brauer 1968, (5G) and (5H)], we have

$$
h(\chi)=0 \Longleftrightarrow m_{\chi \chi}^{z} \equiv 1(\bmod 2) \Longleftrightarrow \sum_{\varphi \in \operatorname{IBr}\left(b_{z}\right)} d_{\chi \varphi}^{z} \equiv 1(\bmod 2) \text {. }
$$

This gives $k_{0}(B) \in\{8,12,16\}$ according to the last column of $D^{z}$. By [Broué 1980, Proposition 1], we also have $h(\chi)=0 \Longleftrightarrow d_{\chi \varphi_{i}}^{u_{i}} \equiv 1(\bmod 2)$ for $i=1,2,3$ where $\operatorname{IBr}\left(b_{u_{i}}\right)=\left\{\varphi_{i}\right\}$. Since the norm of these nonmajor columns is 16 , we have the 
following possibilities for the nonvanishing entries according to $k_{0}(B)$ : sixteen \pm 1 ; twelve \pm 1 and one \pm 2 ; eight \pm 1 and two \pm 2 ; or seven \pm 1 and one \pm 3 .

Taking this together, we can enumerate all the possibilities for the decomposition numbers of nontrivial subsections with GAP. Then the ordinary decomposition matrix (up to multiplication with an invertible matrix) can be determined as the orthogonal space. Finally the square of the ordinary decomposition matrix is the Cartan matrix $C$ of $B$. Now we determine the elementary divisors of $C$ by considering the lower defect groups.

By [Brauer 1969, (7G)], the multiplicity $m(d)$ of the elementary divisor $d \in \mathbb{N}$ of $C$ is given by

$$
m(d)=\sum_{R \in \mathscr{R}} m_{B}^{(1)}(R)
$$

where $\mathscr{R}$ is a set of representatives for the $G$-conjugacy classes of subgroups of $G$ of order $d$. After combining this with [Broué and Olsson 1986, Formula (2S)], we get

$$
m(d)=\sum_{\left(R, b_{R}\right) \in \mathscr{R}^{\prime}} m_{B}^{(1)}\left(R, b_{R}\right)
$$

where $\mathscr{R}^{\prime}$ is a set of representatives for the $G$-conjugacy classes of $B$-subpairs $\left(R, b_{R}\right)$ such that $R$ has order $d$. We have to emphasize that in contrast to other papers we regard $b_{R}$ as a block of $\mathrm{C}_{G}(R)$ instead of $R \mathrm{C}_{G}(R)$. Let $b_{D}$ be a Brauer correspondent of $B$ in $\mathrm{C}_{G}(D)$. Then after changing the representatives if necessary, we may assume $\left(R, b_{R}\right) \leq\left(D, b_{D}\right)$ for $\left(R, b_{R}\right) \in \mathscr{R}^{\prime}$. Then it is well-known that $b_{R}$ is uniquely determined by $R$. Since the fusion of these subpairs is controlled by $\mathrm{N}_{G}\left(D, b_{D}\right)$, we get

$$
m(d)=\sum_{R \in \mathscr{R}^{\prime \prime}} m_{B}^{(1)}\left(R, b_{R}\right)
$$

where $\mathscr{R}^{\prime \prime}$ is a set of representatives for the $\operatorname{Aut}_{\mathscr{F}}(D)$-conjugacy classes of subgroups of $D$ of order $d$.

It is well-known that we have $m(32)=1$. Now we discuss smaller values for $d$. We begin with the case $d=2$. For this, let $m_{B}^{(1)}\left(Q, b_{Q}\right)>0$ for some $Q$ with $|Q|=2$. Then $\left(Q, b_{Q}\right)$ is in fact a subsection and 2 is also an elementary divisor of the Cartan matrix of $b_{Q}$. In particular, $l\left(b_{Q}\right)>1$. This shows that $Q=\mathrm{Z}(D)$. One can show that 2 occurs as elementary divisor of $C_{z}$ exactly four times. If we apply the same arguments to the block $b_{z}$ instead of $B$, we see that $m(2)=m_{B}^{(1)}\left(Q, b_{Q}\right)=4$.

Now let $2<d<32$ and $Q \leq D$ such that $|Q|=d$. Then by [Broué and Olsson 1986, (2Q)], we have $m_{B_{Q}}^{(1)}(Q)>0$ where $B_{Q}:=b_{Q}^{\mathrm{N}_{G}\left(Q, b_{Q}\right)}$. Since $Q$ is fully $\mathscr{F}_{-}$ normalized, [Linckelmann 2006, Theorem 2.4] implies that $\mathrm{C}_{D}(Q)$ is a defect group of $b_{Q}$ and $\mathrm{N}_{D}(Q)$ is a defect group of $B_{Q}$. By [An 2011, Proposition 2.1], also the block $b_{Q}$ is controlled. If we follow the proof of this proposition more 
closely, it turns out that $\left(\mathrm{C}_{D}(Q), b_{Q \mathrm{C}_{D}(Q)}\right)$ is a Sylow $b_{Q}$-subpair. So the inertial quotient of $b_{Q}$ is

$$
\begin{aligned}
\mathrm{N}_{\mathrm{C}_{G}(Q)}\left(\mathrm{C}_{D}(Q), b_{Q \mathrm{C}_{D}(Q)}\right) / \mathrm{C}_{D}(Q) \mathrm{C}_{\mathrm{C}_{G}(Q)}\left(\mathrm{C}_{D}(Q)\right) \\
\quad \leq \mathrm{N}_{G}\left(Q \mathrm{C}_{D}(Q), b_{Q \mathrm{C}_{D}(Q)}\right) \cap \mathrm{C}_{G}(Q) / \mathrm{C}_{D}(Q) \mathrm{C}_{G}\left(Q \mathrm{C}_{D}(Q)\right) .
\end{aligned}
$$

All odd-order automorphisms of

$$
\operatorname{Aut}_{\mathscr{F}}\left(Q \mathrm{C}_{D}(Q)\right)=\mathrm{N}_{G}\left(Q \mathrm{C}_{D}(Q), b_{Q \mathrm{C}_{D}(Q)}\right) / \mathrm{C}_{G}\left(Q \mathrm{C}_{D}(Q)\right)
$$

come from restrictions of $\operatorname{Aut}_{\mathscr{F}}(D)$. However, the automorphism of order 5 in $\operatorname{Aut}_{\mathscr{F}}(D)$ cannot centralize $Q$ since $2<d$. Hence, the inertial index of $b_{Q}$ is 1 and $l\left(b_{Q}\right)=1$. Finally, [Olsson 1980, Theorem 5.11] and the remark following it show

$$
1=l\left(b_{Q}\right) \geq m_{B_{Q}}^{(1)}(Q)+m_{B_{Q}}^{(1)}\left(\mathrm{N}_{D}(Q)\right)=m_{B_{Q}}^{(1)}(Q)+1
$$

and $m_{B_{Q}}^{(1)}(Q)=0$. Taking these arguments together, we proved that the elementary divisors of $C$ are 32, 2, 2, 2, 2, 1, ., 1 (including the possibility of no 1 at all).

Using this, our GAP program reveals that the only possibility for the generalized decomposition numbers is

$$
\left(\begin{array}{rrrrrrrrrrrrr}
1 & 1 & 1 & 1 & . & . & . & . & . & . & . & . & . \\
1 & 1 & . & . & 1 & 1 & . & . & . & . & . & . & . \\
1 & 1 & . & . & . & . & 1 & 1 & . & . & . & . & . \\
1 & 1 & . & . & . & . & . & . & 1 & 1 & . & . & . \\
1 & . & 1 & . & 1 & . & 1 & . & 1 & . & 1 & 1 & 1 \\
-1 & . & . & 1 & . & 1 & . & 1 & . & 1 & 3 & -1 & -1 \\
-1 & . & . & 1 & . & 1 & . & 1 & . & 1 & -1 & 3 & -1 \\
-1 & . & . & 1 & . & 1 & . & 1 & . & 1 & -1 & -1 & 3
\end{array}\right)^{\mathrm{T}}
$$

(up to permutations and choosing signs as described earlier). In particular, $k(B)=13$, $k_{0}(B)=8$ and $l(B)=5$. Moreover, $C$ is uniquely determined up to basic sets. Hence, $C=C_{z}$ up to basic sets because in case $z \in \mathrm{Z}(G), B$ and $b_{z}$ would coincide. It remains to determine $k_{i}(B)$ for $i>0$. For this, let $\psi \in \operatorname{Irr}(B)$ be the fourth character in the numbering above. In particular, $\psi$ has height 0 . Then for a character $\chi \in \operatorname{Irr}(B)$ with $h(\chi)>0$, we can see that $m_{\chi \psi}^{z}$ is divisible by 4 but not by 8 . Thus, [Brauer 1968, $(5 \mathrm{H})$ ] implies $k_{2}(B)=5$.

For the defect group in Proposition 4.3, the inertial index could also be 3. However, in this case the computational effort is too big.

In [Sambale 2011c], we verified Brauer's $k(B)$-conjecture for defect groups of order at most 32 but not isomorphic to the extraspecial group $D_{8} * D_{8}$. We are finally able to handle this remaining group as well. 
Theorem 4.4. Brauer's $k(B)$-conjecture holds for defect groups with a central cyclic subgroup of index at most 16 . In particular, the $k(B)$-conjecture holds for the 2-blocks of defect at most 5.

Proof. Let $B$ be a $p$-block with defect group $D$ of the stated form. By [Sambale $2011 \mathrm{c}$, Theorems 1 and 3], we may assume that there is a major $B$-subsection $\left(z, b_{z}\right)$ such that $D /\langle z\rangle \cong C_{2}^{4}$ (in particular, $p=2$ ) and $B$ has inertial index 9. We apply [Feit 1982, Theorem V.9.17]. For this, it suffices to determine the Cartan matrix of $b_{z}$ (only up to basic sets). Thus, we may consider a 2-block $B$ with elementary abelian defect group $D$ of order 16 and inertial index 9. As in [Külshammer and Sambale 2013, Lemma 2.2], we obtain a list of possible Cartan matrices of $B$. However, since we are considering $9 \times 9$ matrices it is very hard to see if two of these candidates only differ by basic sets. In order to reduce the set of possible Cartan matrices further, we apply various ad hoc matrix manipulations as permutations of rows and columns and elementary row/column operations. After this procedure, we end up with a list of only ten possible Cartan matrices of $B$ that might be all equal up to basic sets. For the purpose of illustrating, we display one of these matrices:

$$
\left(\begin{array}{rrrrrrrrr}
4 & -1 & 1 & . & 1 & 1 & 2 & . & . \\
1 & 4 & . & 1 & -1 & 1 & . & 1 & 1 \\
1 & . & 4 & 1 & -1 & 1 & 2 & -1 & -1 \\
. & 1 & 1 & 4 & . & . & . & 2 & . \\
1 & -1 & -1 & . & 4 & . & 1 & 1 & 1 \\
1 & 1 & 1 & . & . & 4 & 1 & 1 & 1 \\
2 & . & 2 & . & 1 & 1 & 4 & . & -2 \\
. & 1 & -1 & 2 & 1 & 1 & . & 4 & . \\
. & 1 & -1 & . & 1 & 1 & -2 & . & 4
\end{array}\right) .
$$

It can be seen that all diagonal entries are 4 (for every one of these ten matrices). In order to apply [Feit 1982, Theorem V.9.17], let $C$ be one of these ten matrices. Then we have a positive definite integral quadratic form $q$ corresponding to the matrix $16 C^{-1}$. We need to find the minimal nonzero value of $q$ among all integral vectors. More precisely, we have to check if a value strictly smaller than 9 is assumed by $q$. By [Liebeck 1971, Theorem 1], it suffices to consider only vectors with entries in $\{0, \pm 1\}$ (observe that the notation of a quadratic form given by a matrix is the same in [Feit 1982] and [Liebeck 1971]). Hence, there are only $3^{9}$ values to consider. An easy computer computation shows that in fact the minimum of $q$ is at least 9 . So Brauer's $k(B)$-conjecture follows from [Feit 1982, Theorem V.9.17].

We like to point out that we do not know a single Cartan matrix such that Brauer's $k(B)$-conjecture would not follow from [Héthelyi et al. 2013, Theorem 2.4] or from [Feit 1982, Theorem V.9.17]. Since these two results are somehow related, it seems 
interesting to investigate the following problem: Let $C=\left(c_{i j}\right) \in \mathbb{Z}^{l \times l}$ be the Cartan matrix of a $p$-block with defect $d$. Assume that for all integral, positive definite quadratic forms $q\left(x_{1}, \ldots, x_{l\left(b_{u}\right)}\right)=\sum_{1 \leq i \leq j \leq l} q_{i j} x_{i} x_{j}$ we have

$$
\sum_{1 \leq i \leq j \leq l} q_{i j} c_{i j}>p^{d}
$$

Then prove that $x p^{d} C^{-1} x^{\mathrm{T}} \geq l$ for all $0 \neq x \in \mathbb{Z}^{l}$. If this can be done, the $k(B)$ conjecture would follow in full generality. A diagonal matrix shows that this argument fails for arbitrary positive definite, symmetric matrices $C$.

In the next proposition, we take a closer look at the defect group $D_{8} * D_{8}$.

Proposition 4.5. Let $B$ be a block of a finite group $G$ with defect group $D \cong D_{8} * D_{8}$. Suppose that the inertial quotient $\operatorname{Out}_{\mathscr{F}}(D)$ has order 3 and acts freely on $D / \Phi(D)$. Then $k(B)=11, k_{0}(B)=8$ and $l(B)=3$. Moreover, the Cartan matrix of $B$ is given by

$$
2\left(\begin{array}{lll}
2 & 1 & 1 \\
1 & 2 & 1 \\
1 & 1 & 6
\end{array}\right)
$$

up to basic sets. For the numbers $k_{i}(B)(i \geq 1)$, we have the following cases: $\left(k_{1}(B), k_{2}(B)\right) \in\{(0,3),(2,1)\}$.

Proof. Let $\mathscr{F}$ be the fusion system of $B$. By [Stancu 2006, Theorem 5.3], $\mathscr{F}$ is controlled by $\operatorname{Aut}_{\mathscr{F}}(D)$. By hypothesis, $\operatorname{Out}_{\mathscr{F}}(D) \cong C_{3}$ acts freely on $D / \Phi(D)$. Hence, there are two major and five nonmajor subsections. The Cartan matrix of the nontrivial major subsection $\left(z, b_{z}\right)$ is given by

$$
2\left(\begin{array}{lll}
2 & 1 & 1 \\
1 & 2 & 1 \\
1 & 1 & 6
\end{array}\right)
$$

up to basic sets. In particular, $k(B) \leq 16$. The nonmajor subsections $\left(u, b_{u}\right)$ all satisfy $l\left(b_{u}\right)=1$. Since $B$ is centrally controlled, we have $k(B) \geq 11$. The first two columns of the $b_{z}$ decomposition numbers have the form

$$
\left(\begin{array}{llllllllllll}
1 & 1 & 1 & 1 & . & . & \ldots & . & . & \cdots & \cdots & . \\
1 & 1 & . & . & 1 & 1 & \ldots & . & . & . & \cdots & .
\end{array}\right)^{\mathrm{T}}
$$

up to signs and permutations (compare with the proof of Proposition 4.3). For the third column, we have essentially 17 possibilities, which we do not list explicitly here. As in Proposition 4.3, we get $k_{0}(B) \in\{8,12,16\}$ and also the positions of the characters of height 0 depending on the third column of $D_{z}$. Since every element of order 4 in $D$ is conjugate to its inverse, all generalized decomposition numbers are integers. For each of the 17 cases, we proceed by enumerating the five columns of 
nonmajor subsections with the help of a computer. Entirely similar to the proof of Proposition 4.3, we see that the elementary divisors of the Cartan matrix of $B$ are $32,2,2,1, \ldots, 1$. Now the computations reveal $k(B)=11, k_{0}(B)=8, l(B)=3$ and the Cartan matrix of $B$ up to basic sets. However, the value of $k_{1}(B)$ does not follow immediately from these calculations. Instead we obtain the two cases $\left(k_{1}(B), k_{2}(B)\right) \in\{(0,3),(2,1)\}$.

It is easy to construct examples for Proposition 4.5 such that $k_{1}(B)=0$. In contrast, $k_{1}(B)=2$ would contradict the ordinary weight conjecture (see [Robinson 2004]).

The next proposition concerns the Sylow 2-subgroup of $\operatorname{PSU}(3,4)$ as mentioned above. This will result will be used in an upcoming diploma thesis.

Proposition 4.6. Let $B$ be a block of a finite group $G$ with inertial index 15 and defect group $D \in \operatorname{Syl}_{2}(\mathrm{PSU}(3,4))$. Then the elementary divisors of the Cartan matrix of $B$ lie in $\{1,4,64\}$, where 4 occurs with multiplicity at most 4 .

Proof. Since $D$ is a Suzuki 2-group, [Craven and Glesser 2012, Theorem 4.4] tells us that the fusion system $\mathscr{F}$ of $B$ is controlled. So as in the proof of Proposition 4.3, the multiplicity of $d$ as an elementary divisor of the Cartan matrix $C$ of $B$ is given by

$$
m(d)=\sum_{R \in \mathscr{R}^{\prime \prime}} m_{B}^{(1)}\left(R, b_{R}\right),
$$

where $\mathscr{R}^{\prime \prime}$ is a set of representatives for the $\operatorname{Aut}_{\mathscr{F}}(D)$-conjugacy classes of subgroups of $D$ of order $d$. Assume first $d=2$ and $m_{B}^{(1)}\left(Q, b_{Q}\right)>0$ for $|Q|=2$. Then $\left(Q, b_{Q}\right)$ is a subsection and $Q \subseteq \mathrm{Z}(D)$. One can show that $b_{Q}$ has defect group $D$ and inertial index 5. Moreover, $b_{Q}$ covers a block $\overline{b_{Q}}$ of $\mathrm{C}_{G}(Q) / Q$ with defect group $D / Q \cong D_{8} * Q_{8}$. Hence, Proposition 4.3 implies that all elementary divisors of the Cartan matrix of $b_{Q}$ are divisible by 4 . This contradiction shows that $m(2)=0$. Now suppose that $2<d<64$. Again we assume $m_{B}^{(1)}\left(Q, b_{Q}\right)>0$ such that $|Q|=d$. We argue as in the proof of Proposition 4.3. The inertial quotient of $b_{Q}$ is given by

$$
\begin{aligned}
\mathrm{N}_{\mathrm{C}_{G}(Q)}\left(\mathrm{C}_{D}(Q), b_{Q \mathrm{C}_{D}(Q)}\right) / \mathrm{C}_{D}(Q) \mathrm{C}_{\mathrm{C}_{G}(Q)}\left(\mathrm{C}_{D}(Q)\right) \\
\quad \leq \mathrm{N}_{G}\left(Q \mathrm{C}_{D}(Q), b_{Q \mathrm{C}_{D}(Q)}\right) \cap \mathrm{C}_{G}(Q) / \mathrm{C}_{D}(Q) \mathrm{C}_{G}\left(Q \mathrm{C}_{D}(Q)\right) .
\end{aligned}
$$

Every odd order automorphism in

$$
\mathrm{N}_{G}\left(Q \mathrm{C}_{D}(Q), b_{Q \mathrm{C}_{D}(Q)}\right) / \mathrm{C}_{G}\left(Q \mathrm{C}_{D}(Q)\right)=\operatorname{Aut}_{\mathscr{F}}\left(Q \mathrm{C}_{D}(Q)\right)
$$

comes from a restriction of $\operatorname{Aut}_{\mathscr{F}}(D)$. Moreover, $\operatorname{Out}_{\mathscr{F}}(D)$ acts freely on $D / \Phi(D)$. So in case $d>4$, we see that these odd order automorphisms cannot lie in $\mathrm{C}_{G}(Q)$. Hence, in this case $l\left(b_{Q}\right)=1$ and $m(d)=0$ (compare with Proposition 4.3). It remains to deal with the case $Q=\mathrm{Z}(D)=\Phi(D)$. Then we have $b_{Q}=b_{D}^{\mathrm{C}_{G}(\mathrm{Z}(D))}$. 
Moreover, $b_{Q}$ has defect group $D$ and inertial index 5. Looking at the covered block of $\mathrm{C}_{G}(Q) / Q$, we see that $l\left(b_{Q}\right)=5$. Hence,

$$
5=l\left(b_{Q}\right) \geq m_{B_{Q}}^{(1)}(Q)+m_{B_{Q}}^{(1)}\left(\mathrm{N}_{D}(Q)\right)=m_{B_{Q}}^{(1)}(Q)+1
$$

by [Olsson 1980, Theorem 5.11] and the remark following it. This gives $m(4)=$ $m_{B_{Q}}^{(1)}(Q) \leq 4$, and the proof is complete.

Our next result handles rather unknown groups of order 32. The key observation here is that the fusion system is constrained and thus quite easy to understand.

Proposition 4.7. Let $B$ be a nonnilpotent block of a finite group $G$ with defect group $D \cong \operatorname{SmallGroup}(32, q)$ for $q \in\{28,29\}$. Then $k(B)=14, k_{0}(B)=8$, $k_{1}(B)=6$ and $l(B)=2$.

Proof. Let $\mathscr{F}$ be the fusion system of $B$. Using GAP, one can show that $\operatorname{Aut}(D)$ is a 2group. In particular, $\operatorname{Out}_{\mathscr{F}}(D)=1$. Moreover, one can show using general results in [Sambale 2012b] that $D$ contains only one $\mathscr{F}$-essential subgroup $Q$. Here $C_{2}^{2} \times C_{4} \cong$ $Q \unlhd D$. In particular, $\mathscr{F}$ is constrained. Another GAP calculation shows that $\mathscr{F}$ is the fusion system of the groups $\operatorname{SmallGroup}(96,187)$ or $\operatorname{SmallGroup}(96,185)$ for $q \in\{28,29\}$, respectively. We have ten $B$-subsections up to conjugation. The center of $D$ is a four-group and $\Phi(Q) \subseteq \mathrm{Z}(D)$. Hence, an odd order automorphism of $Q$ cannot act on $\mathrm{Z}(D)$. It follows that we have four major subsections $(1, B),\left(z, b_{z}\right)$, $\left(v, b_{v}\right)$ and $\left(w, b_{w}\right)$ up to conjugation. Here we may assume that $l\left(b_{v}\right)=l\left(b_{w}\right)=1$. On the other hand, $b_{z}$ dominates a nonnilpotent block with defect group $D /\langle z\rangle \cong$ $D_{8} \times C_{2}$. Thus, by [Sambale 2011c, Proposition 3] we have $l\left(b_{z}\right)=2$. Also we find an element $u \in Q$ such that $b_{u}$ is nonnilpotent with defect group $Q$. Here [Sambale 2011c, Proposition 2] implies $l\left(b_{u}\right)=3$. The remaining nonmajor subsections split in one subsection $\left(u_{1}, b_{1}\right)$ of defect 16 and four subsections $\left(u_{i}, b_{i}\right)(i=2,3,4,5)$ of defect 8. Here $l\left(b_{i}\right)=1$ for $i=1, \ldots, 5$. In particular, Olsson's conjecture $k_{0}(B) \leq 8=\left|D: D^{\prime}\right|$ follows at once. Since $B$ is centrally controlled, we also obtain $l(B) \geq 2$ and $k(B) \geq 14$. So the generalized decomposition numbers $d_{i j}^{v}$ consist of eight entries \pm 1 and six entries \pm 2 . Hence, $k(B)=14, k_{0}(B)=8, k_{1}(B)=6$ and $l(B)=2$.

Also in the next proposition, the corresponding fusion system is easy to understand since it is controlled. Another advantage here is that $k(B)$ is relatively small so that the computational effort is small as well.

Proposition 4.8. Let $D$ be a central cyclic extension of $\operatorname{SmallGroup}(32, q)$ for $q \in\{33,34\}$. Then Brauer's $k(B)$-conjecture holds for all blocks with defect group $D$.

Proof. It suffices to consider a block $B$ with defect group $D \cong \operatorname{SmallGroup}(32, q)$ for $q \in\{33,34\}$ as usual. GAP shows that $B$ is a controlled block with inertial index 3. Hence, the fusion system of $B$ is the same as the fusion system of the 
group $D \rtimes C_{3}$. It follows that there are only six $B$-subsections up to conjugation; two of them are major. For $1 \neq z \in \mathrm{Z}(D)$, we have $l\left(b_{z}\right)=1$. Let us denote the four nonmajor subsections by $\left(u_{i}, b_{i}\right)$ for $i=1, \ldots, 4$. We may assume that $b_{1}$ has defect group $C_{2}^{3}$. It is easy to see that $\operatorname{Aut}_{\mathscr{F}}(D)$ restricts to the inertial group of $b_{1}$. In particular, $l\left(b_{1}\right)=e\left(b_{1}\right)=3$. Moreover, the Cartan matrix of $b_{1}$ is given by

$$
2\left(\begin{array}{lll}
2 & 1 & 1 \\
1 & 2 & 1 \\
1 & 1 & 2
\end{array}\right)
$$

up to basic sets (see [Sambale 2011b, Theorem 3]). Moreover, $b_{2}$ has defect 3 and $b_{3}$ and $b_{4}$ have defect 4. Here, $l\left(b_{2}\right)=l\left(b_{3}\right)=l\left(b_{4}\right)=1$. In particular, Olsson's conjecture $k_{0}(B) \leq 8=\left|D: D^{\prime}\right|$ follows. Looking at $d_{i j}^{z}$, we get $k(B) \leq 14$. The numbers $d_{i j}^{u_{1}}$ can certainly be arranged in the form

$$
\left(\begin{array}{lllllllllll}
1 & 1 & 1 & 1 & . & . & . & . & . & \cdots & . \\
1 & 1 & . & . & 1 & 1 & . & . & . & \cdots & . \\
1 & 1 & . & . & . & . & 1 & 1 & . & \cdots & .
\end{array}\right)^{\mathrm{T}}
$$

Using the contributions, it follows that $k_{0}(B)=8$. We can easily add the column for $\left(u_{2}, b_{2}\right)$ as

$$
\left(\begin{array}{lllllllll}
1 & 1 & -1 & \cdots & -1 & 0 & \cdots & 0
\end{array}\right)^{\mathrm{T}} \text { or } \quad\left(\begin{array}{lllllllllll}
1 & -1 & 1 & -1 & 1 & -1 & 1 & -1 & 0 & \cdots & 0
\end{array}\right)^{\mathrm{T}} .
$$

We investigate next the elementary divisors of the Cartan matrix of $B$. For this, we consider the multiplicity of $\left\langle u_{1}\right\rangle$ as a lower defect group. The multiplicity of 2 as an elementary divisor of the Cartan matrix of $b_{1}$ is certainly 2. Since $\left\langle u_{1}\right\rangle$ is the only lower defect group of order 2 of $b_{1}$, we have $m(2)=m_{B}^{(1)}\left(\left\langle u_{1}\right\rangle, b_{1}\right)=$ $m_{b_{1}}^{(1)}\left(\left\langle u_{1}\right\rangle, b_{1}\right)=2$. This shows $l(B) \geq 3$ and $k(B) \geq 10$. Every automorphism of order 3 of $D$ fixes only two elements in $D$. Thus, it follows as in Proposition 4.3 that $m(d)=0$ for $2<d<32$. We have essentially four possibilities for the numbers $d_{i j}^{z}$ :

- eight entries \pm 1 and six entries \pm 2 ,

- eight entries \pm 1 , two entries \pm 2 and one entry \pm 4 ,

- seven entries \pm 1 , four entries \pm 2 and one entry \pm 3 or

- six entries \pm 1 , two entries \pm 2 and two entries \pm 3 .

In particular, $k(B)$ determines $k_{i}(B)$ for $i \geq 1$ uniquely. It remains to add the generalized decomposition numbers corresponding to $\left(u_{3}, b_{3}\right)$ and $\left(u_{4}, b_{4}\right)$. Here the situation is distinguished by $q \in\{33,34\}$. Assume first that $q=34$. Then $u_{3}^{-1}$ and $u_{4}^{-1}$ are conjugate to $u_{3}$ and $u_{4}$, respectively. Hence, the numbers $d_{i j}^{u_{3}}$ and $d_{i j}^{u_{4}}$ are integers. It is easy to see that such a column must consist of the following (nonzero) entries: 
- eight entries \pm 1 and two entries \pm 2 and

- seven entries \pm 1 and one entry \pm 3 .

In contrast, for $q=33$ the elements $u_{3}^{-1}$ and $u_{4}$ are conjugate. So we may assume $u_{4}:=u_{3}^{-1}$, and it suffices to consider the column $d_{i j}^{u_{3}}$ whose entries are Gaussian integers. Let us write $d_{\chi \varphi_{3}}^{u_{3}}:=a(\chi)+b(\chi) i$, where $\operatorname{IBr}\left(b_{3}\right)=\left\{\varphi_{3}\right\}, a, b \in \mathbb{Z}^{k(B)}$ and $i:=\sqrt{-1}$. Then $(a, a)=(b, b)=8$ and $(a, b)=0$. Since we have only one pair of algebraically conjugate subsections, there is only one pair of 2-conjugate characters (see [Feit 1982, Lemma IV.6.10]). This shows that $b$ consists of two entries \pm 2 . Now $k_{0}(B)=8$ implies that $a$ has eight entries \pm 1 .

As usual, we enumerate all these configurations of the generalized decomposition matrix and obtain the Cartan matrix of $B$ as orthogonal space. However, we get two possibilities $l(B) \in\{3,4\}$. We are not able to exclude the case $l(B)=4$ despite its contradiction of Alperin's weight conjecture. Anyway in both cases $l(B) \in\{3,4\}$, all candidates for the Cartan matrix satisfy [Héthelyi et al. 2013, Theorem 2.4]. The claim follows.

We add a short discussion about the defect group

$$
\begin{aligned}
D & :=\operatorname{SmallGroup}(32,27) \\
& \cong\left\langle a, b, c \mid a^{2}=b^{2}=c^{2}=[a, b]=\left[a,{ }^{c} a\right]=\left[{ }^{c} a, b\right]=\left[b,{ }^{c} b\right]=1\right\rangle \cong C_{2}^{4} \rtimes C_{2} .
\end{aligned}
$$

Let $\mathscr{F}$ be a nonnilpotent fusion system on $D$. One can show $Q:=\left\langle a, b,{ }^{c} a,{ }^{c} b\right\rangle \cong C_{2}^{4}$ is the only possible $\mathscr{F}$-essential subgroup. In particular, $\mathscr{F}$ is controlled or constrained (note that controlled is a strong form of constrained). In the controlled case, we have $\mathscr{F}_{F}=\mathscr{F}_{D}\left(D \rtimes C_{3}\right)=\mathscr{F}_{D}(\operatorname{Small} \operatorname{Group}(96,70))$. In the noncontrolled case, we have various possibilities for $\mathscr{F}_{F}$ according to $\operatorname{Out}_{\mathscr{F}}(Q) \in\left\{S_{3}, D_{10}, S_{3} \times C_{3}\right.$, SmallGroup (18,4), $D_{10} \times C_{3}$ \} (see [Sambale 2012b, Lemma 3.11]). These possibilities are represented by the following groups:

$$
\begin{array}{ll}
\text { SmallGroup (96, 195), } & \text { SmallGroup }(288,1025), \\
\text { SmallGroup (96, 227), } & \text { SmallGroup }(288,1026), \\
\text { SmallGroup (160, 234), } & \text { SmallGroup }(480,1188) .
\end{array}
$$

Here observe that in case $\operatorname{Out}_{\mathscr{F}}(Q)=S_{3}$ there are essentially two different actions of $\operatorname{Out}_{\mathscr{F}}(Q)$ on $Q$. The cases $\operatorname{Out}_{\mathscr{F}}(Q) \in\left\{S_{3} \times C_{3}\right.$, $\left.\operatorname{Small} \operatorname{Group}(18,4)\right\}$ also differ by $\operatorname{Out}_{\mathscr{F}}(D) \in\left\{C_{3}, 1\right\}$, respectively. Additionally, for $\operatorname{Out}_{\mathscr{F}}(Q)=\operatorname{SmallGroup}(18,4)$ there exists a nontrivial 2-cocycle on $\operatorname{Out}_{\mathscr{F}}(Q)$ (on the other hand, the Künneth formula implies $\mathrm{H}^{2}\left(S_{3} \times C_{3}, F^{\times}\right)=0$ for an algebraically closed field $F$ of characteristic 2). This gives even more examples for blocks with defect group $D$. For example, a nonprincipal 2-block of $\operatorname{SmallGroup}(864,3996)$ has defect group $D$ and only one irreducible Brauer character. In all these examples, $l(B)$ assumes the 
values $1,2,3,5,6,9$. We will not consider the block invariants in full generality although it might be possible. We also end the discussion about the remaining groups of order 32. In most cases (especially when $9 \times 9$ Cartan matrices show up), the computational effort to compute the corresponding block invariants is too big.

In the following table, we enumerate all groups of order 32 by using the small groups library and give information about blocks with corresponding defect groups. In many cases, it can be shown with GAP that there are no nontrivial fusion systems. These cases were also determined in [van der Waall 1991], but with the enumeration of [Hall and Senior 1964]. Using a conversion between both enumerations provided by Eamonn O'Brien, we confirm the results in [van der Waall 1991]. We denote the modular group of order $2^{n} \geq 16$ by $M_{2^{n}}$, i.e., the unique group of class 2 with a cyclic maximal subgroup.

\begin{tabular}{|c|c|c|c|c|}
\hline small group \# & description & invariants & comments & reference \\
\hline 1 & $C_{32}$ & known & nilpotent & \\
\hline 2 & $\operatorname{MNA}(2,2)$ & known & controlled & $\begin{array}{l}\text { [Sambale 2011a; } \\
\quad \text { Eaton et al. 2012] }\end{array}$ \\
\hline 3 & $C_{8} \times C_{4}$ & known & nilpotent & \\
\hline 4 & $C_{8} \rtimes C_{4}$ & known & nilpotent & [Sambale 2012c] \\
\hline 5 & $\operatorname{MNA}(3,1)$ & known & & [Sambale 2011a] \\
\hline 6 & $\operatorname{MNA}(2,1) \rtimes C_{2}$ & known & nilpotent & GAP \\
\hline 7 & $M_{16} \rtimes C_{2}$ & known & nilpotent & GAP \\
\hline 8 & $C_{2} \cdot \operatorname{MNA}(2,1)$ & known & nilpotent & GAP \\
\hline 9 & $D_{8} \rtimes C_{4}$ & known & bicyclic & Theorem 3.1 \\
\hline 10 & $Q_{8} \rtimes C_{4}$ & known & bicyclic & Theorem 3.3 \\
\hline 11 & $C_{4} \prec C_{2}$ & known & & [Külshammer 1980] \\
\hline 12 & $C_{4} \rtimes C_{8}$ & known & nilpotent & [Sambale 2012c] \\
\hline 13 & $C_{8} \rtimes C_{4}$ & known & nilpotent & [Sambale 2012c] \\
\hline 14 & $C_{8} \rtimes C_{4}$ & known & nilpotent & [Sambale 2012c] \\
\hline 15 & $C_{8} \cdot C_{4}$ & known & nilpotent & [Sambale 2012c] \\
\hline 16 & $C_{16} \times C_{2}$ & known & nilpotent & \\
\hline 17 & $M_{32}$ & known & nilpotent & [Sambale 2012c] \\
\hline 18 & $D_{32}$ & known & maximal class & [Brauer 1974] \\
\hline 19 & $S D_{32}$ & known & maximal class & [Olsson 1975] \\
\hline 20 & $Q_{32}$ & known & maximal class & [Olsson 1975] \\
\hline 21 & $C_{4}^{2} \times C_{2}$ & known & controlled & [Usami 1988] \\
\hline 22 & $\operatorname{MNA}(2,1) \times C_{2}$ & known & constrained & Proposition 4.2 \\
\hline 23 & $\left(C_{4} \rtimes C_{4}\right) \times C_{2}$ & known & nilpotent & GAP \\
\hline 24 & $C_{4}^{2} \rtimes C_{2}$ & known & nilpotent & GAP \\
\hline 25 & $D_{8} \times C_{4}$ & known & & [Sambale 2012a] \\
\hline 26 & $Q_{8} \times C_{4}$ & known & & [Sambale 2013b] \\
\hline 27 & $C_{2}^{4} \rtimes C_{2}$ & & & \\
\hline
\end{tabular}




\begin{tabular}{ccccc} 
small group \# & description & invariants & comments & reference \\
\hline 28 & $\left(C_{4} \times C_{2}^{2}\right) \rtimes C_{2}$ & known & constrained & Proposition 4.7 \\
29 & $\left(Q_{8} \times C_{2}\right) \rtimes C_{2}$ & known & constrained & Proposition 4.7 \\
30 & $\left(C_{4} \times C_{2}^{2}\right) \rtimes C_{2}$ & known & nilpotent & GAP \\
31 & $\left(C_{4} \times C_{4}\right) \rtimes C_{2}$ & known & nilpotent & GAP \\
32 & $C_{2}^{2} \cdot C_{2}^{3}$ & known & nilpotent & GAP \\
33 & $\left(C_{4} \times C_{4}\right) \rtimes C_{2}$ & & controlled & \\
34 & $\left(C_{4} \times C_{4}\right) \rtimes C_{2}$ & & controlled & \\
35 & $C_{4} \rtimes Q_{8}$ & known & nilpotent & GAP \\
36 & $C_{8} \times C_{2}^{2}$ & known & controlled & [Usami 1988] \\
37 & $M_{16} \times C_{2}$ & known & nilpotent & GAP \\
38 & $D_{8} * C_{8}$ & known & & [Sambale 2013a] \\
39 & $D_{16} \times C_{2}$ & known & & [Sambale 2012a] \\
40 & $S D_{16} \times C_{2}$ & known & & [Sambale 2013b] \\
41 & $Q_{16} \times C_{2}$ & known & & [Sambale 2013b] \\
42 & $D_{16} * C_{4}$ & known & & [Sambale 2013a] \\
43 & $\left(D_{8} \times C_{2}\right) \rtimes C_{2}$ & & & \\
44 & $\left(Q_{8} \times C_{2}\right) \rtimes C_{2}$ & & & \\
45 & $C_{4} \times C_{2}^{3}$ & known & controlled & Theorem 4.1 \\
46 & $D_{8} \times C_{2}^{2}$ & & & \\
47 & $Q_{8} \times C_{2}^{2}$ & & controlled & \\
48 & $\left(D_{8} * C_{4}\right) \times C_{2}$ & & controlled & \\
49 & $D_{8} * D_{8}$ & & controlled & \\
50 & $D_{8} * Q_{8}$ & & controlled & \\
51 & $C_{2}^{5}$ & & controlled & \\
& & & &
\end{tabular}

We apply these results to Theorem 2.2.

Theorem 4.9. Let $D$ be a cyclic central extension of one of the following groups:

(i) a metacyclic group,

(ii) a minimal nonabelian group,

(iii) a group of order at most 16,

(iv) $\prod_{i=1}^{n} C_{2^{m_{i}}}$ where $\left|\left\{m_{i}: i=1, \ldots, n\right\}\right| \geq n-1$,

(v) $M \times C$ where $M$ has maximal class and $C$ is cyclic,

(vi) $M * C$ where $M$ has maximal class and $C$ is cyclic,

(vii) $D_{2^{n}} \rtimes C_{2^{m}}, Q_{2^{n}} \rtimes C_{2^{m}}$ and $D_{2^{n}} . C_{2^{m}}$ as in Theorems 3.1, 3.3 and 3.2,

(viii) SmallGroup(32, q) for $q \in\{11,22,28,29,33,34\}$, or

(ix) a group that admits only the nilpotent fusion system.

Then Brauer's $k(B)$-conjecture holds for every 2-block with defect group $D$. 
Proof. The case (iii) follows from Theorem 4.4. In case (viii), the result follows from Propositions 4.2, 4.7 and 4.8 and [Külshammer 1980]. In all other cases, it suffices to show $l(B) \leq 3$ for every block $B$ with defect group given in the remaining list of the statement. For the abelian defect group $\prod_{i=1}^{n} C_{2^{m_{i}}}$ where $\left|\left\{m_{i}: i=1, \ldots, n\right\}\right| \geq n-1$, it is easy to see that the inertial index $e(B)$ is at most 3. Thus, results of Puig and Usami [Usami 1988] imply Alperin's weight conjecture in this case. Now $l(B) \leq 3$ follows easily. For the remaining cases, the claim was shown in [Sambale 2012c; Eaton et al. 2012; Sambale 2011c; 2012a; 2013b; 2013a] and the present paper.

One can show with GAP that Theorem 4.9 suffices to verify Brauer's $k(B)$ conjecture for 244 of the 267 defect groups of order 64 . Here we also use the following elementary observation: Let $z \in \mathrm{Z}(D)$ such that every fusion system on $D /\langle z\rangle$ is controlled. If $\mathrm{C}_{\mathrm{Aut}(D)}(z)$ is a 2-group, then Brauer's $k(B)$-conjecture holds for every block with defect group $D$.

For the group $D \cong \operatorname{SmallGroup}(64,265)$, we can argue even more subtly. Every block $B$ with defect group $D$ fulfills $e(B) \in\{1,3,5\}$. In case $e(B)=3$, we find an element $z \in \mathrm{Z}(D)$ such that $D /\langle z\rangle$ is elementary abelian. Then [Usami 1988] implies $k(B) \leq 64$. On the other hand if $e(B)=5$, we choose $z \in \mathrm{Z}(D)$ such that $D /\langle z\rangle \cong D_{8} * Q_{8}$. Here the $k(B)$-conjecture follows from Proposition 4.3.

For the purpose of further research, we state all indices $q$ such that Brauer's $k(B)$-conjecture for the defect group $\operatorname{SmallGroup}(64, q)$ is not known so far:

$134,135,136,137,138,139,202,224,229,230,231$,

238, 239, 242, 254, 255, 257, 258, 259, 261, 262, 264, 267.

This implies the following corollary:

Corollary 4.10. Let $B$ be a 2-block with defect group $D$ of order at most 64 . If $D$ is generated by two elements, then Brauer's $k(B)$-conjecture holds for $B$.

One can also formulate a version of Theorem 4.9 for $k_{0}(B)$ using Theorem 2.4. Compare also with [Héthelyi et al. 2013, Theorem 2.5].

Corollary 4.11. Let $D$ be a 2-group containing a cyclic subgroup of index at most 4 . Then Brauer's $k(B)$-conjecture holds for every block with defect group $D$.

Proof. We may assume that $D$ is not metacyclic. In particular, $|D| / \exp D=4$. If $D$ is abelian, the result follows from [Sambale 2011c, Corollary 2]. Hence, let us assume that $D$ is nonabelian. Then $D$ is one of the groups given in [Ninomiya 1994, Theorem 2]. We will consider this list of groups case by case and apply Theorem 4.9. We remark that the terms "quasidihedral" and "semidihedral" have different meanings in [Ninomiya 1994].

The group $G_{1}$ is metacyclic. 
For the groups $G_{2}$ and $G_{3}$, we even know the block invariants precisely.

Now consider $G_{4}$. Here the element $a$ lies in the center. In particular, the group is a cyclic central extension of a group of order 4 . The $k(B)$-conjecture follows.

For the group $G_{5}$, the element $b$ lies in the center. Moreover, $G_{5} /\langle b\rangle$ is abelian and has a cyclic subgroup of index 2 . Again the claim holds.

The groups $G_{6}, G_{7}, G_{8}$ and $G_{9}$ are metacyclic.

The groups $G_{10}$ and $G_{11}$ are cyclic central extensions of metacyclic groups.

In $G_{12}$, the subgroup $\langle a\rangle$ is normal; in particular, $a^{2^{m-3}} \in \mathrm{Z}\left(G_{12}\right)$. Moreover, $b$ is central in $G_{12} /\left\langle a^{2^{m-3}}\right\rangle$ and $G_{12} /\left\langle a^{2^{m-3}}\right\rangle \cong D_{2^{m-2}} \times C_{2}$. The claim follows.

In $G_{13}$ and $G_{14}$, we see that $b$ is central and the corresponding quotient is certainly metacyclic.

Next, $a^{2^{m-3}} \in \mathrm{Z}\left(G_{15}\right)$ and $G_{15} /\left\langle a^{2^{m-3}}\right\rangle \cong D_{2^{m-2}} \times C_{2}$. Exactly the same argument applies to $G_{16}$.

For $G_{17}$, we have $c^{-1} a^{2} c=a b a b=a^{2+2^{m-3}}$ and $a^{4} \in \mathrm{Z}\left(G_{17}\right)$. Since $G_{17} /\left\langle a^{4}\right\rangle$ has order 16, the claim follows.

The group $G_{18}$ is slightly more complicated. In general, the core of $\langle a\rangle$ has index at most 8 . Thus, $a^{2^{m-3}}$ is always central (in all of these groups). Adjusting notation slightly gives

$$
G_{18} /\left\langle a^{2^{m-3}}\right\rangle \cong\left\langle a, b, c \mid a^{2^{m-3}}=b^{2}=c^{2}=[a, b]=1, c a c=a^{-1} b\right\rangle .
$$

We define new elements in this quotient by $\tilde{v}:=a^{2} b, \tilde{x}:=b c$ and $\tilde{a}:=a c$. Then $\tilde{v}^{2^{m-4}}=1, \tilde{a}^{2}=b$ and $\tilde{a}^{4}=1$. Moreover, $c b c=c($ acac $) c=b$. It follows that $\tilde{x}^{2}=1$ and $\tilde{x} \tilde{v} \tilde{x}=\tilde{v}^{-1}$. Hence, $\langle\tilde{v}, \tilde{x}\rangle \cong D_{2^{m-3}}$. Now $\tilde{a} \tilde{v} \tilde{a}^{-1}=c a^{2} b c=a^{-2} b=\tilde{v}^{-1}$ and finally $\tilde{a} \tilde{x} \tilde{a}^{-1}=a^{2} c=\tilde{v} \tilde{x}$. Since $G_{18} /\left\langle a^{2^{m-3}}\right\rangle=\langle\tilde{v}, \tilde{x}, \tilde{a}\rangle$, we see that this precisely the group from Theorem 3.1. The claim follows.

The groups $G_{19}, G_{20}$ and $G_{21}$ are metacyclic.

In $G_{22}$, the element $a^{4}$ is central and $G_{22} /\left\langle a^{4}\right\rangle$ has order 16 .

Let us consider $G_{23}$. As above, we have

$$
G_{23} /\left\langle a^{2^{m-3}}\right\rangle \cong\left\langle a, b, c \mid a^{2^{m-3}}=b^{2}=c^{2}=[a, b]=1, c a c=a^{-1+2^{m-4}} b\right\rangle
$$

(observe that the relation $[b, c] \equiv 1\left(\bmod \left\langle a^{2^{m-3}}\right\rangle\right)$ follows from $\left.b \equiv a^{1+2^{m-4}} c a c\right)$. Here we define $\tilde{v}:=a^{2+2^{m-4}} b, \tilde{x}:=b c$ and $\tilde{a}:=a c$. Then again $\langle\tilde{v}, \tilde{x}\rangle \cong D_{2^{m-3}}$. Moreover, $\tilde{a}^{2}=a^{2^{m-4}} b, \tilde{a}^{4}=1$ and $\tilde{a} \tilde{x} \tilde{a}^{-1}=b c a^{-1} c a c=a^{2+2^{m-4}} c=\tilde{v} \tilde{x}$. So $G_{23} /\left\langle a^{2^{m-3}}\right\rangle$ is the group from Theorem 3.1.

Now it is easy to see that $G_{24} /\left\langle a^{2^{m-3}}\right\rangle \cong G_{25} /\left\langle a^{2^{m-3}}\right\rangle \cong G_{23} /\left\langle a^{2^{m-3}}\right\rangle$.

Finally the group $G_{26}$ has order 32 , so also here the $k(B)$-conjecture holds. This completes the proof.

For every integer $n \geq 6$, there are exactly 33 groups of order $2^{n}$ satisfying the hypothesis of Corollary 4.11 . 


\section{Olsson's conjecture}

We have seen in [Héthelyi et al. 2013] that Olsson's conjecture holds for all controlled 2-blocks of defect at most 5. Using the table above, we remove the controlled condition.

Theorem 5.1. Olsson's conjecture holds for all 2-blocks of defect at most 5.

Proof. By the remark above it, suffices to consider only the defect groups $D:=$ $\operatorname{SmallGroup}(32, m)$ where $m \in\{27,43,44,46\}$. Let $B$ be a block with defect group $D$ and fusion system $\mathscr{F}$. Then we can find (with GAP) an element $u \in D$ such that $\left|\mathrm{C}_{D}(u)\right|=\left|D: D^{\prime}\right|$. Moreover, we can choose $u$ such that every element $v \in D$ of the same order also satisfies $\left|\mathrm{C}_{D}(u)\right|=\left|D: D^{\prime}\right|$. Hence, the subgroup $\langle u\rangle$ is fully $\mathscr{F}$-centralized. In particular, $\mathrm{C}_{D}(u)$ is a defect group of the block $b_{u}$. Now the claim follows from [Héthelyi et al. 2013, Proposition 2.5(ii)].

In [Héthelyi et al. 2013], we also verified Olsson's conjecture for defect groups of $p$-rank 2 provided $p>3$. We use the opportunity to explore the case $p=3$ in more detail.

Theorem 5.2. Let $B$ be a 3-block of a finite group $G$ with defect group D. Assume that D has 3-rank 2 but not maximal class. Then Olsson's conjecture holds for $B$.

Proof. By [Héthelyi et al. 2013, Theorem 5.6], we may assume that the fusion system $\mathscr{F}$ of $B$ is not controlled. Then $|D| \geq 3^{4}$ since $D$ does not have maximal class. By [Díaz et al. 2007, Theorems 4.1 and 4.2], it remains to handle the groups $D=G(3, r ; \epsilon)$ of order $3^{r}$ where $r \geq 5$ and $\epsilon \in\{ \pm 1\}$ as in [Díaz et al. 2007, Theorem 4.7] (by [Díaz et al. 2007, Remark A.3], $G(3,4 ; \epsilon)$ has maximal class). Assume that $D$ is given by generators and relations as in Theorem A.1 of the same paper. Consider the element $x:=a c$. By [Díaz et al. 2007, Lemma A.8], $x$ is not contained in the unique $\mathscr{F}$-essential ( $\mathscr{F}$-Alperin) subgroup $C(3, r-1)=\left\langle a, b, c^{3}\right\rangle$.

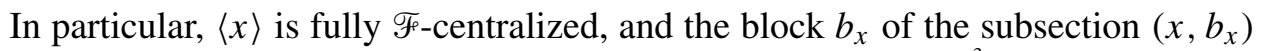
has defect group $\mathrm{C}_{D}(x)$. It is easy to see that $D^{\prime}=\left\langle b, c^{3^{r-3}}\right\rangle \cong C_{p} \times C_{p}$. It follows that $x^{3^{r-4}} \equiv c^{3^{r-4}} \neq \equiv 1\left(\bmod D^{\prime}\right)$ and $|\langle x\rangle| \geq 3^{r-3}$. As usual, we have $\left|\mathrm{C}_{D}(x)\right| \geq\left|D: D^{\prime}\right|=3^{r-2}$. In case $\left|\mathrm{C}_{D}(x)\right| \geq 3^{r-1}$, we get the contradiction $b \in D^{\prime} \subseteq \mathrm{C}_{D}(x)$. Hence, $\left|\mathrm{C}_{D}(x)\right|=\left|D: D^{\prime}\right|$ and $\mathrm{C}_{D}(x) /\langle x\rangle$ is cyclic. Now Olsson's conjecture for $B$ follows from [Héthelyi et al. 2013, Proposition 2.5].

Theorem 5.3. Let $B$ be a 3-block of a finite group with defect group $D$ of order at least $3^{4}$. Assume that $D$ has maximal class but is not isomorphic to the group

$$
\begin{gathered}
B(3, r ; 0,0,0)=\left\langle s, s_{1}, \ldots, s_{r-1}\right| s^{3}=s_{r-2}^{3}=s_{r-1}^{3}=\left[s_{1}, s_{2}\right]=\cdots=\left[s_{1}, s_{r-1}\right] \\
\left.\quad=s_{1}^{3} s_{2}^{3} s_{3}=\cdots=s_{r-3}^{3} s_{r-2}^{3} s_{r-1}=1, s_{i}=\left[s_{i-1}, s\right] \quad \text { for } i=2, \ldots, r-1\right\rangle
\end{gathered}
$$

of order $3^{r}$. Then Olsson's conjecture holds for $B$. 
Proof. By [Héthelyi et al. 2013, Theorem 5.6], we may assume that the fusion system $\mathscr{F}$ of $B$ is not controlled. Then $\mathscr{F}$ is given as in [Díaz et al. 2007, Theorem 5.10]. In particular, $D=B(3, r ; 0, \gamma, 0)$ is given by generators and relations as in [Díaz et al. 2007, Theorem A.2] where $\gamma \in\{1,2\}$. Let $D_{1}$ be as in [Huppert 1967, Definition III.14.3]. Observe that in the notation of [Díaz et al. 2007; Blackburn 1958] we have $D_{1}=\gamma_{1}(D)$. From [Díaz et al. 2007, Proposition A.9] we see that $x:=s s_{1} \notin D_{1}$. Moreover, we have $x^{3} \neq 1$ also by the same proposition. Then by [Díaz et al. 2007, Lemma A.15], $x$ does not lie in one of the centric subgroups $D_{1}$, $E_{i}$ or $V_{i}$ for $i \in\{-1,0,1\}$. This shows that $x$ is not $\mathscr{F}$-conjugate to an element in $D_{1}$. By [Huppert 1967, Satz III.14.17], $D$ is not an exceptional group. In particular, [Huppert 1967, Hilfssatz III.14.13] implies $\left|C_{D}(y)\right|=9=\left|D: D^{\prime}\right|$ for all $y \in D \backslash D_{1}$. Hence, $\langle x\rangle$ is fully $\mathscr{F}$-centralized. Thus, the block $b_{x}$ of the subsection $\left(x, b_{x}\right)$ has defect group $\mathrm{C}_{D}(x)$. Now Olsson's conjecture follows from [Héthelyi et al. 2013, Proposition 2.5].

We remark that the method in Theorem 5.3 does not work for the groups $B(3, r ; 0,0,0)$. For example, every block of a subsection of the principal 3-block of ${ }^{3} D_{4}(2)$ has defect at least 3 (here $r=4$ ). However, $\left|D: D^{\prime}\right|=3^{2}$ for every 3 -group of maximal class.

\section{Acknowledgments}

This work is supported by the German Academic Exchange Service (DAAD) and the German Research Foundation (DFG) and was written partly in Santa Cruz, USA. I thank the University of California for its hospitality.

\section{References}

[Alperin et al. 1973] J. L. Alperin, R. Brauer, and D. Gorenstein, "Finite simple groups of 2-rank two”, Scripta Math. 29:3-4 (1973), 191-214. MR 53 \#5728 Zbl 0274.20021

[An 2011] J. An, "Controlled blocks of the finite quasisimple groups for odd primes", Adv. Math. 227:3 (2011), 1165-1194. MR 2012f:20023 Zbl 1247.20008

[Berkovich and Janko 2008] Y. Berkovich and Z. Janko, Groups of prime power order, II, de Gruyter Expositions in Mathematics 47, de Gruyter, 2008. MR 2009m:20026b Zbl 1168.20002

[Blackburn 1958] N. Blackburn, “On a special class of p-groups”, Acta Math. 100 (1958), 45-92. MR 21 \#1349 Zbl 0083.24802

[Brauer 1968] R. Brauer, "On blocks and sections in finite groups, II", Amer. J. Math. 90 (1968), 895-925. MR 39 \#5713 Zbl 0174.05401

[Brauer 1969] R. Brauer, "Defect groups in the theory of representations of finite groups", Illinois J. Math. 13 (1969), 53-73. MR 40 \#248 Zbl 0167.29801

[Brauer 1974] R. Brauer, “On 2-blocks with dihedral defect groups”, pp. 367-393 in Symposia Mathematica, XIII (Rome, 1972), Academic Press, London, 1974. MR 50 \#7315 Zbl 0288.20010 
[Broué 1980] M. Broué, "On characters of height zero", pp. 393-396 in The Santa Cruz Conference on Finite Groups (Santa Cruz, CA, 1979), edited by B. Cooperstein and G. Mason, Proc. Sympos. Pure Math. 37, Amer. Math. Soc., Providence, R.I., 1980. MR 82g:20021 Zbl 0475.20009

[Broué and Olsson 1986] M. Broué and J. B. Olsson, "Subpair multiplicities in finite groups", J. Reine Angew. Math. 371 (1986), 125-143. MR 87j:20026 Zbl 0596.20009

[Craven and Glesser 2012] D. A. Craven and A. Glesser, "Fusion systems on small p-groups", Trans. Amer. Math. Soc. 364:11 (2012), 5945-5967. MR 2946938 Zbl 06199346

[Díaz et al. 2007] A. Díaz, A. Ruiz, and A. Viruel, "All p-local finite groups of rank two for odd prime p", Trans. Amer. Math. Soc. 359:4 (2007), 1725-1764. MR 2008a:20037 Zbl 1113.55010

[Eaton 2003] C. W. Eaton, "Generalisations of conjectures of Brauer and Olsson", Arch. Math. (Basel) 81:6 (2003), 621-626. MR 2004i:20012 Zbl 1046.20007

[Eaton and Moretó 2013] C. W. Eaton and A. Moretó, "Extending Brauer's height zero conjecture to blocks with nonabelian defect groups", Int. Math. Res. Not. (2013), Art. ID rnt131.

[Eaton et al. 2012] C. W. Eaton, B. Külshammer, and B. Sambale, "2-blocks with minimal nonabelian defect groups, II”, J. Group Theory 15:3 (2012), 311-321. MR 2920888 Zbl 1253.20008

[Erdmann 1990] K. Erdmann, Blocks of tame representation type and related algebras, Lecture Notes in Mathematics 1428, Springer, Berlin, 1990. MR 91c:20016 Zbl 0696.20001

[Feit 1982] W. Feit, The representation theory of finite groups, North-Holland Mathematical Library 25, North-Holland, Amsterdam, 1982. MR 83g:20001 Zbl 0493.20007

[GAP 2012] GAP - Groups, Algorithms, and Programming, Version 4.5.6, The GAP Group, 2012, Available at http://www.gap-system.org.

[Gorenstein 1968] D. Gorenstein, Finite groups, Harper \& Row, New York, 1968. MR 38 \#229 Zbl 0185.05701

[Hall and Senior 1964] M. Hall, Jr. and J. K. Senior, The groups of order $2^{n}(n \leq 6)$, Macmillan, New York, 1964. MR 29 \#5889 Zbl 0192.11701

[Héthelyi et al. 2013] L. Héthelyi, B. Külshammer, and B. Sambale, "A note on Olsson's Conjecture", preprint, 2013, Available at https://www.minet.uni-jena.de/algebra/personen/sambale/olssoncon.pdf. To appear in J. Algebra.

[Huppert 1967] B. Huppert, Endliche Gruppen, I, Grundlehren der Math. Wissenschaften 134, Springer, Berlin, 1967. MR 37 \#302 Zbl 0217.07201

[Kessar 2007] R. Kessar, "Introducton to block theory", pp. 47-77 in Group representation theory, edited by M. Geck et al., EPFL Press, Lausanne, 2007. MR 2008f:20020 Zbl 1165.20007

[Kessar and Malle 2013] R. Kessar and G. Malle, "Quasi-isolated blocks and Brauer's height zero conjecture”, Ann. of Math. (2) 178:1 (2013), 321-384. MR 3043583 Zbl 06190562

[Kessar et al. 2012] R. Kessar, S. Koshitani, and M. Linckelmann, "Conjectures of Alperin and Broué for 2-blocks with elementary abelian defect groups of order 8", J. Reine Angew. Math. 671 (2012), 85-130. MR 2983198 Zbl 06100515

[Külshammer 1980] B. Külshammer, "On 2-blocks with wreathed defect groups", J. Algebra 64:2 (1980), 529-555. MR 81i:20008 Zbl 0442.20012

[Külshammer and Okuyama 2000] B. Külshammer and T. Okuyama, "On centrally controlled blocks of finite groups", 2000. unpublished.

[Külshammer and Sambale 2013] B. Külshammer and B. Sambale, "The 2-blocks of defect 4", Represent. Theory 17 (2013), 226-236. MR 3048571 Zbl 06183358

[Liebeck 1971] H. Liebeck, "The location of the minimum of a positive definite integral quadratic form”, J. London Math. Soc. (2) 3 (1971), 477-484. MR 44 \#1193 Zbl 0211.07801 
[Linckelmann 2006] M. Linckelmann, "Simple fusion systems and the Solomon 2-local groups", J. Algebra 296:2 (2006), 385-401. MR 2006i:20024 Zbl 1097.20014

[Linckelmann 2007] M. Linckelmann, "Introduction to fusion systems", pp. 79-113 in Group representation theory, edited by M. Geck et al., EPFL Press, Lausanne, 2007. MR 2008f:20021 Zbl 1161.20007

[Malle and Navarro 2006] G. Malle and G. Navarro, "Inequalities for some blocks of finite groups", Arch. Math. (Basel) 87:5 (2006), 390-399. MR 2007i:20073 Zbl 1109.20011

[Murai 2000] M. Murai, “On subsections of blocks and Brauer pairs”, Osaka J. Math. 37:3 (2000), 719-733. MR 2001k:20018 Zbl 0969.20008

[Nagao and Tsushima 1989] H. Nagao and Y. Tsushima, Representations of finite groups, Academic Press, Boston, 1989. MR 90h:20008 Zbl 0673.20002

[Ninomiya 1994] Y. Ninomiya, "Finite $p$-groups with cyclic subgroups of index $p^{2}$ ", Math. J. Okayama Univ. 36 (1994), 1-21. MR 96h:20044 Zbl 0838.20017

[Oliver and Ventura 2009] B. Oliver and J. Ventura, "Saturated fusion systems over 2-groups", Trans. Amer. Math. Soc. 361:12 (2009), 6661-6728. MR 2010i:20018 Zbl 1244.20015

[Olsson 1975] J. B. Olsson, "On 2-blocks with quaternion and quasidihedral defect groups", $J$. Algebra 36:2 (1975), 212-241. MR 51 \#13016 Zbl 0314.20009

[Olsson 1980] J. B. Olsson, "Lower defect groups", Comm. Algebra 8:3 (1980), 261-288. MR 81g: 20024 Zbl 0426.20008

[Olsson 1981] J. B. Olsson, "Inequalities for block-theoretic invariants", pp. 270-284 in Representations of algebras (Puebla, 1980), edited by M. Auslander and E. Lluis, Lecture Notes in Math. 903, Springer, Berlin, 1981. MR 83i:20013 Zbl 0478.20008

[Rédei 1947] L. Rédei, “Das „schiefe Produkt“ in der Gruppentheorie”, Comment. Math. Helv. 20 (1947), 225-264. MR 9,131a Zbl 0035.01503

[Robinson 1991] G. R. Robinson, "On the number of characters in a block", J. Algebra 138:2 (1991), 515-521. MR 92h:20022a Zbl 0727.20010

[Robinson 1992] G. R. Robinson, “On Brauer's k(B) problem”, J. Algebra 147:2 (1992), 450-455. MR 93b:20020 Zbl 0796.20006

[Robinson 2004] G. R. Robinson, "Weight conjectures for ordinary characters", J. Algebra 276:2 (2004), 761-775. MR 2005c:20021 Zbl 1121.20007

[Robinson 2008] G. R. Robinson, "On the number of characters in a block and the Brauer-Feit matrix", 2008. unpublished.

[Sambale 2011a] B. Sambale, "2-blocks with minimal nonabelian defect groups", J. Algebra 337 (2011), 261-284. MR 2012d:20019 Zbl 1247.20010

[Sambale 2011b] B. Sambale, "Cartan matrices and Brauer's k(B)-conjecture", J. Algebra 331 (2011), 416-427. MR 2012c:20023 Zbl 1230.20010

[Sambale 2011c] B. Sambale, "Cartan matrices and Brauer's k(B)-conjecture, II", J. Algebra 337 (2011), 345-362. MR 2012e:20018 Zbl 1243.20013

[Sambale 2012a] B. Sambale, "Blocks with defect group $D_{2^{n}} \times C_{2^{m}}$ ", J. Pure Appl. Algebra 216:1 (2012), 119-125. MR 2012f:20031 Zbl 1241.20013

[Sambale 2012b] B. Sambale, "Fusion systems on bicyclic 2-groups", preprint, 2012. arXiv 1206. 2962v1

[Sambale 2012c] B. Sambale, "Fusion systems on metacyclic 2-groups", Osaka J. Math. 49:2 (2012), 325-329. MR 2945751 Zbl 1247.20025 
[Sambale 2013a] B. Sambale, "Blocks with central product defect group $D_{2^{n}} * C_{2^{m}}$ ", Proc. Amer. Math. Soc. 141:12 (2013), 4057-4069. MR 3105851 Zbl 06218137

[Sambale 2013b] B. Sambale, "Blocks with defect group $Q_{2^{n}} \times C_{2^{m}}$ and $S D_{2^{n}} \times C_{2^{m}}$ ", Algebr. Represent. Theory 16:6 (2013), 1717-1732. MR 3127355

[Stancu 2006] R. Stancu, "Control of fusion in fusion systems", J. Algebra Appl. 5:6 (2006), 817-837. MR 2007j:20025 Zbl 1118.20020

[Usami 1988] Y. Usami, "On p-blocks with abelian defect groups and inertial index 2 or 3, I", J. Algebra 119:1 (1988), 123-146. MR 89i:20024 Zbl 0659.20008

[van der Waall 1991] R. W. van der Waall, "On p-nilpotent forcing groups”, Indag. Math. (N.S.) 2:3 (1991), 367-384. MR 93b:20040 Zbl 0749.20004

[Watanabe 1991] A. Watanabe, "Notes on p-blocks of characters of finite groups", J. Algebra 136:1 (1991), 109-116. MR 92i:20016 Zbl 0725.20008

Communicated by David Benson

Received 2012-09-30 Revised 2012-10-16 Accepted 2013-03-23

benjamin.sambale@uni-jena.de Mathematisches Institut, Friedrich-Schiller-Universität, D-07737 Jena, Germany 


\section{Algebra \& Number Theory}

msp.org/ant

\section{EDITORS}

MANAGING EDITOR

Bjorn Poonen

Massachusetts Institute of Technology

Cambridge, USA

\author{
EDITORIAL BOARD CHAIR \\ David Eisenbud \\ University of California \\ Berkeley, USA
}

\section{BOARD OF EDITORS}

Georgia Benkart

Dave Benson

Richard E. Borcherds

John H. Coates

J-L. Colliot-Thélène

Brian D. Conrad

Hélène Esnault

Hubert Flenner

Edward Frenkel

Andrew Granville

Joseph Gubeladze

Roger Heath-Brown

Ehud Hrushovski

Craig Huneke

Mikhail Kapranov

Yujiro Kawamata

János Kollár

Yuri Manin

Barry Mazur

Philippe Michel
University of Wisconsin, Madison, USA

University of Aberdeen, Scotland

University of California, Berkeley, USA

University of Cambridge, UK

CNRS, Université Paris-Sud, France

University of Michigan, USA

Freie Universität Berlin, Germany

Ruhr-Universität, Germany

University of California, Berkeley, USA

Université de Montréal, Canada

San Francisco State University, USA

Oxford University, UK

Hebrew University, Israel

University of Virginia, USA

Yale University, USA

University of Tokyo, Japan

Princeton University, USA

Northwestern University, USA

Harvard University, USA

École Polytechnique Fédérale de Lausanne
Susan Montgomery

Shigefumi Mori

Raman Parimala

Jonathan Pila

Victor Reiner

Karl Rubin

Peter Sarnak

Joseph H. Silverman

Michael Singer

Vasudevan Srinivas

J. Toby Stafford

Bernd Sturmfels

Richard Taylor

Ravi Vakil

Michel van den Bergh

Marie-France Vignéras

Kei-Ichi Watanabe

Efim Zelmanov

Shou-Wu Zhang
University of Southern California, USA

RIMS, Kyoto University, Japan

Emory University, USA

University of Oxford, UK

University of Minnesota, USA

University of California, Irvine, USA

Princeton University, USA

Brown University, USA

North Carolina State University, USA

Tata Inst. of Fund. Research, India

University of Michigan, USA

University of California, Berkeley, USA

Harvard University, USA

Stanford University, USA

Hasselt University, Belgium

Université Paris VII, France

Nihon University, Japan

University of California, San Diego, USA

Princeton University, USA

PRODUCTION

production@msp.org

Silvio Levy, Scientific Editor

See inside back cover or msp.org/ant for submission instructions.

The subscription price for 2013 is US $\$ 200 /$ year for the electronic version, and $\$ 350 /$ year $(+\$ 40$, if shipping outside the US) for print and electronic. Subscriptions, requests for back issues and changes of subscribers address should be sent to MSP.

Algebra \& Number Theory (ISSN 1944-7833 electronic, 1937-0652 printed) at Mathematical Sciences Publishers, 798 Evans Hall \#3840, c/o University of California, Berkeley, CA 94720-3840 is published continuously online. Periodical rate postage paid at Berkeley, CA 94704, and additional mailing offices.

ANT peer review and production are managed by EditFLOW ${ }^{\circledR}$ from Mathematical Sciences Publishers.

\section{PUBLISHED BY}

- mathematical sciences publishers

nonprofit scientific publishing

http://msp.org/

(C) 2013 Mathematical Sciences Publishers 


\section{Algebra \& Number Theory}

Volume $7 \quad$ No. $9 \quad 2013$

Multiplicities associated to graded families of ideals Steven Dale Cutkosky

Normal coverings of linear groups

JOHN R. BRITNELL and ATTILA MARÓTI

Modularity of the concave composition generating function

George E. ANDrews, Robert C. RhOAdes and SANDER P. ZWEgERS

ANDREW NILES

Regular permutation groups of order $m p$ and Hopf Galois structures

2203

ТIMOTHY KOHL

Further evidence for conjectures in block theory

2241

BENJAMIN SAMBALE

Network parametrizations for the Grassmannian

2275

Kelli TALASKa and LAUREN Williams

Chow quotients of toric varieties as moduli of stable log maps

2313

Qile Chen and Matthew Satriano

Vinberg's representations and arithmetic invariant theory

JACK A. THORNE 